\title{
Initial-value predictability of prominent modes of North Pacific subsurface temperature in a CGCM
}

\author{
Haiyan Teng • Grant Branstator
}

Received: 29 September 2009/ Accepted: 19 January 2010/Published online: 31 March 2010

(C) Springer-Verlag 2010

\begin{abstract}
Three 40-member ensemble experiments and a 700 year control run are used to study initial value predictability in the North Pacific in Community Climate System Model version 3 (CCSM3). Our focus is on the leading two empirical orthogonal functions (EOFs) of subsurface temperature variability, which together produce an eastward propagating mode. Predictability is measured by relative entropy, which compares both the mean and spread of predictions of ensembles to the model's climatological distribution of states. Despite the fact that EOF1, which is structurally similar to the observational Pacific Decadal Oscillation (PDO), has pronounced spectral peaks on decadal time scales, its predictability is less than 6 years. Additional predictability resides in the tendency of EOF1 to evolve to EOF2, primarily through simple advective processes. The propagating mode represented by the combination of EOF1 and EOF2 is predictable for about a decade. Information in both the mean and spread of predicted ensembles contribute to this predictability. Among the leading 15 EOFs, EOF1 is the least predictable mode in terms of the rate at which the corresponding principal component disperses in the ensemble experiments. However, it can produce enhanced predictability of the whole system by inducing EOF2, which is one of the two EOFs with the slowest dispersion rate. The first two EOFs can also enhance the ensemble mean (or "signal") component of predictability of the entire system. For typical amplitude initial states, this component contributes to predictability for about 6 years. For initial states with unusually high amplitude projections onto these two EOFs,
\end{abstract}

H. Teng $(\bowtie) \cdot$ G. Branstator

National Center for Atmospheric Research, Boulder, CL, USA

e-mail: hteng@ucar.edu this contribution can last much longer. The major findings from the three ensemble experiments are replicated and generalized when the initial condition predictability for each of many hundreds of different initial states is estimated. These estimates are derived from the behavior of a linear inverse model (LIM) that is based on the intrinsic variability present in the control run.

\section{Introduction}

While models, observations and assimilation methods have now progressed to the stage that attempts are being made to carry out forecasts on decadal timescales (Smith et al. 2007, Keenlyside et al. 2008), it remains unclear to what degree information in the initial state can add to the skill of such forecasts. For the climate system is chaotic and its evolution could be so sensitive to inevitable errors in the initial state that information residing there is lost after only a few years. If so, there is no point in trying to predict beyond this range unless one is also taking into account changing external forcing.

In this paper, we contribute to the quantification of the initial-value predictability of the coupled atmosphereocean system by concentrating on the predictability of prominent intrinsic modes of decadal variability. We focus on these large scale features because they have high amplitude and thus have the potential to impact weather and climate events that affect society. Furthermore, in studies of weather prediction (e.g. Tribbia 1988 and Branstator et al. 1993) and intraseasonal variability (e.g. Newman et al. 2003a), prominent modes have been found to be more predictable than other features, and we are interested in whether this may also be true for decadal timescales. 
Given the short climate record, the only way to estimate decadal predictability limits resulting from initial state uncertainty is to use models. Most previous model-based studies have focused on the North Atlantic, particularly the Atlantic meridional overturning circulation (AMOC; Griffies and Bryan 1997a, 1997b; Collins 2002; Collins and Sinha 2003; Pohlmann et al. 2004; Collins et al. 2006b). Many studies concur that the AMOC is potentially predictable a decade in advance, but the characteristics of the AMOC, including its predictability limit, vary from model to model (Hurrell et al. 2009). And it remains controversial whether the AMOC can bring decadal predictability to the surface climate.

In the North Pacific, the thermohaline circulation is too weak to be responsible for the pronounced decadal variability observed in sea surface temperature (SST). Instead, about one-third of the SST decadal variability is associated with the "Pacific Decadal Oscillation", or PDO (Mantua et al. 1997), a mode captured by the first empirical orthogonal function (EOF) of SST. Because of its importance, much of our investigation is concentrated on the predictability of the PDO. Several mechanisms have been proposed for the PDO (Miller and Schneider 2000; Seager et al. 2001), and they have different implications for its predictability. Latif and Barnett $(1994,1996)$ hypothesized that enhanced predictability can result from the oscillatory nature of the PDO, which according to their analysis relies on coupling between the subtropical ocean gyre circulation and the Aleutian low pressure system. On the other hand, driving of the ocean by white weather noise alone, without two-way coupling, can produce decadal variability in the ocean (Frankignoul and Hasselmann 1977; Frankignoul et al. 1997). A third scenario that has been suggested emphasizes the ocean circulation and slow dynamical oceanic modes that contribute to a reddening of the SST signals, even when the ocean-to-atmosphere feedback is weak (Saravanan and McWilliams 1998).

Predictability from initial conditions (what Lorenz (1963) refers to as "predictability of the first kind") is measured by determining for how long the predicted distribution of an ensemble of similar initial states is distinguishable from the climatological distribution. Hence asking how predictable the PDO is on decadal time scales is different from asking how much variability it has on these scales. Even if its temporal spectrum has a decadal peak, if fast growing errors resulting from initial uncertainties quickly conceal the PDO signals in forecasts, then the PDO is not predictable. Note measuring predictability is different from measuring a quantity that some refer to as "diagnostic potential predictability" (Boer 2000). This quantity measures the variability on, say, decadal time scales that is in excess of that expected if the part of the spectrum for frequencies lower than annual were white.
The straightforward way to quantitatively measure predictability is to use ensemble experiments with perturbed initial conditions under the "perfect model" assumption. Most studies use the ensemble spread as measured by standard deviations to quantify its limit, and predictability is lost when the spread is indistinguishable from the climatological spread.

Earlier studies have found that the answer to whether there is decadal predictability in the North Pacific varies with the analysis method and the variable that is considered. If one assumes there is a correspondence between diagnostic potential predictability and predictability, some modeling studies find there is decadal predictability of SST in some regions of the North Pacific (Boer 2004). On the other hand, others using stochastic modeling methods to estimate predictability, have found that SST is predictable for only 1-2 years in both the observational record (Alexander et al. 2008) and AOGCMs (Newman 2007). SST anomalies can persist into the following winter due to the "reemergence mechanism", where the anomalies stay below the mixed layer in summer and re-entrained into the mixed layer in the following fall and winter (Deser et al. 2003). But even with the influence of this effect, most studies conclude North Pacific SSTs are unpredictable on decadal time scales.

For decadal predictions of the ocean, the emphasis on SST in many studies is natural because it is through SST that the ocean affects the atmosphere. Although SST does not appear to be predictable beyond 1 or 2 years in most regions, enhanced predictability may exist in multi-year averaged SST. In addition to temporally averaged SST, depth averaged temperature has been found to have extended predictability (Griffies and Bryan 1997a). Depth averaging serves as an implicit temporal filter that minimizes the effects of daily weather fluctuations. As an example of this effect consider Fig. 1a. It shows the average root mean square difference of North Pacific ocean variables between all pairs of realizations of a 40-member ensemble of integrations performed with Community Climate System Model version 3 (CCSM3). In these integrations, which are described in more detail in Sect. 2, the initial states are identical except for the atmosphere. The spread in upper $300 \mathrm{~m}$ temperature grows much slower than the spread of SST. From a domain average point of view, it takes about 10 years for the North Pacific subsurface temperature to diverge to random states, in contrast to less than 5 years in SST.

This result indicates there may exist decadal predictability in the subsurface temperature in the North Pacific in CCSM3, but it does not explain what processes and structures this predictability is associated with. In particular it does not address whether it may arise from some dynamic modes such as the PDO. With this figure in mind, 

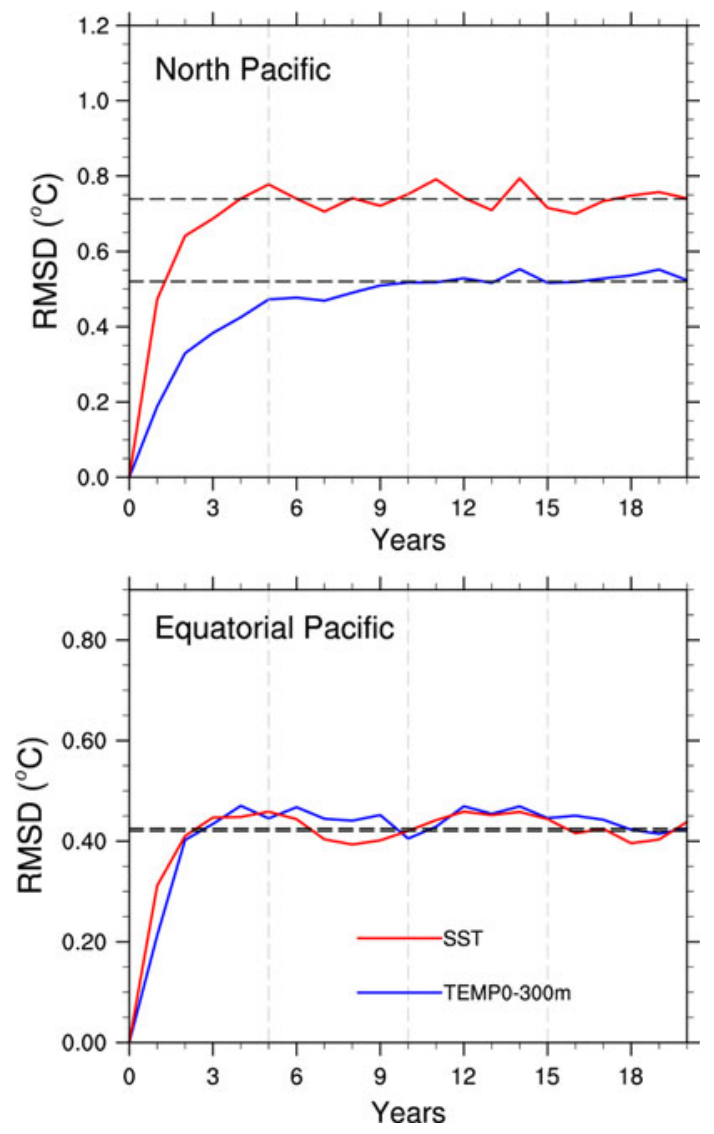

Fig. 1 Root mean square difference (RMSD) in SST and upper$300 \mathrm{~m}$ temperature between pairs of members from a 40-member ensemble simulation that differ only in the atmospheric initial condition (later referred to as Ensemble I, see Sect. 2a for more details) in the North Pacific (top panel, $120^{\circ} \mathrm{E}-110^{\circ} \mathrm{W}, 20^{\circ} \mathrm{N}-65^{\circ} \mathrm{N}$ ) and the equatorial Pacific (bottom panel, $120^{\circ} \mathrm{E}-80^{\circ} \mathrm{W}, 20^{\circ} \mathrm{S}-20^{\circ} \mathrm{N}$ ). The color curves are RMSD averaged across the 780 pairs that are present in the 40-member ensemble. Black dashed lines correspond to 95\% of the average RMSD between two random states in the CCSM3 control run

in this paper we will address the following questions: to what extent is there predictability in the prominent modes in the subsurface temperature? How much do the prominent modes contribute to the predictability of the entire North Pacific region?

To answer these questions we will first identify strong modes of North Pacific subsurface decadal variability in CCSM3 using EOF analysis. It turns out that the leading EOF is similar to the structure of the PDO. Then we will quantify the predictability of these patterns using both ensemble experiments, including the one used to produce Fig. 1, as well as a 700 year control run of that model. In addition to focusing on the role of intrinsic modes in decadal prediction, this work expands on previous studies by examining the upper $300 \mathrm{~m}$ temperature instead of SST, and by analyzing a model whose predictability has not been previously quantified. Moreover, instead of treating the
PDO as a standing mode represented by a single pattern, we find the leading two EOFs in upper $300 \mathrm{~m}$ temperature in CCSM3 represent different phases of a propagating mode making it more physical and instructive to consider the predictability of this pair of structures in combination than individually. One method we employ that facilitates the quantification of the predictability limit of a mode of this type is to use relative entropy (Kleeman 2002) from information theory.

To describe our investigation, this paper is organized as follows. Section 2 introduces CCSM3, the ensemble experiments, and analysis methods. The prominent modes in the subsurface temperature in CCSM3 as well as the processes that contribute to their behavior are described in Sect. 3. Initial value predictability of the dominant modes is estimated using three ensemble experiments in Sect. 4. In order to test whether the ensemble experiment results reflect general properties of CCSM3, we see whether similar predictability properties for these modes are implied by the intrinsic variability of that model in a long control run. These predictability properties are determined by construction and analysis of a linear inverse model in Sect. 5. How the leading modes contribute to the predictability of North Pacific subsurface temperatures in general is discussed in Sect. 6, followed by a summary and discussion of our study's implications in Sect. 7.

\section{Model, experiments and analysis methods}

\subsection{CCSM3 and experiments}

CCSM3 is a fully coupled model that includes four components: atmosphere, ocean, land, and sea ice (Collins et al. 2006a). These components are linked via a flux coupler and no flux corrections are employed. Alexander et al. (2006) have compared the extratropical atmosphere-ocean variability over the Northern Hemisphere in a CCSM3 control run to observations. In the North Pacific, the model simulates the PDO reasonably well. The largest discrepancy is that the PDO lacks the connection to tropical Pacific SST that is seen in the observations. Since tropical Pacific SST can strongly influence the atmospheric circulation of the midlatitudes through the atmospheric bridge (Alexander et al. 2002), the model may underestimate climate anomalies associated with the PDO over the surrounding continents. Because the main purpose here is to quantify the predictability of the prominent modes in the North Pacific rather than their impacts on variables over land, such model weaknesses may not be important. On the other hand, since apparent tropical-midlatitude interactions implied by the observational record on decadal time scales are not well understood it is possible that these interactions have an 
influence on the predictability of the PDO (Newman et al. 2003 b) that is not present in the simulations we study here.

To assess the predictability of prominent modes in the North Pacific, we assume CCSM3 is a perfect surrogate for nature and perform three 40-member ensemble experiments using a configuration with a T42 atmosphere and a nominal $1^{\circ}$ ocean. Each of the three experiments has a different initial state while the realizations in each experiment differ from each other in one of two ways. Ensemble I is branched from January 1st of year 2000 of a 20th century historical run. Its 40 initial conditions are identical for land, ocean, and sea ice gridpoints, but atmospheric initial conditions are unique to each realization; they are taken from different days in December 1999 and January 2000 of the historical run. Ensembles II and III are branched from two of the realizations of Ensemble I in year 2008. In the latter two ensembles, all 40 members share the same initial states for all four model components; each realization is distinct only because the solar constant is slightly different in each one. The realization-to-realization variations in the solar constant are so small as to produce no appreciable effects on the climate of each realization. On the other hand, after one time step these variations have produced very small variations in the atmosphere and thus act to set each realization off on a different trajectory. Hence the solar constant perturbations serve the same purpose as would small perturbations to the initial atmospheric state.

In our study of the predictability of prominent North Pacific modes, an inevitable question is whether the predictability limit of these modes is sensitive to their initial amplitudes. This question guides our choice of initial ocean states. As mentioned in the introduction and as described in detail later, the mode our study primarily focuses on is defined by the leading two EOFs of subsurface temperature. The initial state for Ensemble I has weak projections on both these patterns. By contrast, the initial state for Ensemble II is chosen because it features a very strong projection onto EOF1 but has a very weak projection on EOF2, and the initial state of Ensemble III has a very strong projection on EOF2 but a very weak projection on EOF1. Ensemble $I$ is integrated for 62 years, but in results not presented here, we have found that if there is any predictable signal beyond year 20 it is very weak. Therefore, we only consider the first 20 years of that ensemble, and Ensembles II and III are integrated for only 20 years.

Although our study focuses on initial-value predictability of intrinsic modes, A1B scenario forcing (Meehl et al. 2006) has been included in all three ensembles so that these experiments can also be used for assessing predictability resulting from this external forcing (Lorenz's (1963) "predictability of the second kind"). In a following-up study, we will compare predictability of both kinds using these ensemble experiments. In the present study, we assume intrinsic variability is independent of the trend caused by the external A1B forcing trend. This allows us to concentrate on intrinsic variability by first calculating the 40-member averaged linear trend in Ensemble I during 1999-2061 at each grid point, and then removing this trend from each realization in all three ensembles. The residuals are the variability whose predictability we examine.

In addition to the three ensembles, we employ a 1000 year control run (Bryan et al. 2006) for learning about the model's intrinsic modes, for constructing the inverse model describe below, and for assessing statistical significance. It is identical to the model used for the ensemble experiments except the forcing is set to the conditions for 1990. Our study only uses the last 700 years of the control run to avoid the years when spin-up occurs.

Our analysis uses values of SST and mean-upper-300 m temperature in the North Pacific region defined by $\left(20^{\circ} \mathrm{N}-\right.$ $\left.65^{\circ} \mathrm{N}, 120^{\circ} \mathrm{E}-110^{\circ} \mathrm{W}\right)$. We average the ocean temperature in the top 16 layers of the ocean model and then regrid it from the native ocean grids to the atmospheric model's T42 grid. All results we present are derived from annual mean data. We have also tested the use of DJF means, and it does not affect the main results of our study.

\subsection{Relative entropy}

In predictability studies the most commonly employed measures of whether and by how much a forecast distribution differs from the climatological distribution use some indication of the spread of the ensemble prediction, e.g. the root mean square difference (RMSD) in Fig. 1. A more comprehensive measure determines how much information is provided by the prediction over and above the information one has from knowledge of climatology. For an $\mathrm{n}$-dimensional system with state vector $\left(x_{1}, x_{2}, \ldots, x_{n}\right)$ and climatological probability distribution $c$, the additional information given by a perfect prediction with distribution $e$ is

$R=\int_{-\infty}^{\infty} \ldots \int_{-\infty}^{\infty} \int_{-\infty}^{\infty} e \ln \left(\frac{e}{c}\right) d x_{1} d x_{2}, \ldots, d x_{n}$

In information theory, $R$ is called the relative entropy (Kleeman 2002). Relative entropy can be thought of as being a distance between distributions $e$ and $c$ though strictly speaking it is not a distance in the mathematical sense. For predictions of a Markov process, $R$ always decreases monotonically with time provided complete state vectors are employed. When $R$ asymptotes to a value of zero, it indicates that the two distributions are identical, and the initial-value predictability is lost. 
If we assume both $e$ and $c$ are Gaussian with the first and second moments denoted by $\mu_{e}, \sigma_{e}^{2}$ and $\mu_{c}, \sigma_{c}^{2}$, respectively, relative entropy in Eq. 1 can be expressed as:

$$
\begin{aligned}
R= & \frac{1}{2}\left\{\ln \left[\frac{\operatorname{det}\left(\sigma_{c}^{2}\right)}{\operatorname{det}\left(\sigma_{e}^{2}\right)}\right]+\operatorname{tr}\left[\left(\sigma_{e}^{2}\left(\sigma_{c}^{2}\right)^{-1}\right]\right.\right. \\
& \left.+\left(\mu_{e}-\mu_{c}\right)^{T}\left(\sigma_{c}^{2}\right)^{-1}\left(\mu_{e}-\mu_{c}\right)-n\right\} .
\end{aligned}
$$

Later, we will refer to the first two terms minus $n$ as the dispersion component and the third term as the signal component of the relative entropy.

In our study, we have 700 samples from the control run to estimate the climatological distribution, but only 40 samples for predictions. The limited sample size of the prediction ensembles induces an error in the estimation of $R$. Because $R$ is positive definite this error has a bias, and $R$ asymptotes to a non-zero value when the predictability is lost. We estimate this value by calculating $R$ from many samples of 40 randomly chosen states from the control run.

Relative entropy has several advantages compared to a conventional indication of predictability based only on the spread of a single variable (e.g. the ratio of predicted to climatological variance). First, it can take into account how both the predicted mean and spread compare to the corresponding climatological quantities. Clearly, this is a worthwhile attribute since there is still useful information if a prediction indicates a shift of the mean, even if it has the same spread as climatology. Second, it can quantify predictability of a system that has more than one degree of freedom. For our study, this attribute is important because we wish to consider predictability of modes that have more than one degree of freedom.

\subsection{LIM}

The CCSM3 ensemble approach has one important limitation, namely, predictability limits revealed by an ensemble experiment may be only valid for the particular ocean/land/ice initial states used in the experiment. In order to test whether the results we find in our ensemble experiments reflect more general predictability properties of CCSM3, we construct a linear inverse model (LIM) based on the control run. The resulting LIM should approximate the dynamical properties of CCSM3 provided the underlying assumption of the LIM is valid. This assumption is that the system being represented can be well approximated by a linear system driven by Gaussian white noise. Once we have approximated CCSM3 in this way, we can estimate its predictability limits for very many initial states.

Here we provide only a brief description of the LIM formulation, as LIMs have been described in detail in many papers since being introduced to atmospheric science by
Penland(1989). Assume the dynamical system of interest can be well approximated by

$\frac{d x}{d t}=\mathbf{B} x+\xi$,

where $\mathbf{x}$ is the departure of the state of the system from its mean, $\mathbf{B}$ is a linear feedback operator and $\xi$ is Gaussian white noise. Then $\mathbf{B}$ can be found from observations of the system because

$\exp \left(\mathbf{B} \tau_{0}\right)=\mathbf{C}\left(\tau_{0}\right) \mathbf{C}^{-1}(0)$

where $\mathbf{C}(\tau)$ is the lag- $\tau$ covariance matrix of $x$ and $\tau_{0}$ is the particular lag used to calculate $\mathbf{B}$. (Also the covariance $\mathbf{Q}$ of the driving noise can be derived from $\mathbf{B}$ and $C(0)$ via a Lyapunov equation though we do not need $\mathbf{Q}$ for our LIM applications).

When using CCSM3 to investigate predictability we study the evolution of an ensemble of similar initial states under the influence of chaotic dynamics. In the LIM approximation the ensemble is replaced by a continuous distribution that evolves from a single initial state under the influence of the noise. Now if $\mathbf{G}(\tau)$ is the lag- $\tau$ propagator of Eq. 3, i.e.

$\mathbf{G}(\tau)=\exp (\mathbf{B} \tau)=\left[\mathbf{G}\left(\tau_{0}\right)\right]^{\tau / \tau_{0}}$,

then the distribution of forecasts at range $\tau$ for initial state $\tilde{x}(t)$ has mean

$\bar{x}(t+\tau)=\mathbf{G}(\tau) \tilde{x}(t)$

and covariance

$\left\langle x(t+\tau) x^{T}(t+\tau)\right\rangle=\mathbf{C}(0)-\mathbf{G}(\tau) \mathbf{C}(0) \mathbf{G}^{T}(\tau)$,

where \langle\rangle represents an average over the distribution. Assuming CCSM3 statistics are stationary, $\mathbf{G}(\tau)$ based on CCSM3 data will converge to zero for large $\tau$, and so the distribution of LIM forecast states will take on the covariance structure of CCSM3's climate and their mean will be zero. So, as is true for the full system, at some forecast range the predictability of forecasts from a LIM based on CCSM3 will vanish.

We measure the predictability of a distribution of LIM forecasts just as we do a CCSM3 ensemble, namely in terms of relative entropy using the mean and standard deviation from Eq. 6 and 7. One property of LIM forecasts, as with any linear stochastic oscillator, is that the noise characteristics are independent of the initial state. Therefore, as can be seen in Eq. 7, the covariance structure of a forecast is not affected by the initial state. Hence only the signal component of relative entropy in Eq. 2 varies with the initial condition, and an initial state with a particular structure will have higher predictability the larger its amplitude is. 
In order to reduce data processing and to exclude patterns of variability whose amplitudes are weak, we use the leading 15 EOFs in the upper- $300 \mathrm{~m}$ temperature in the North Pacific to represent the entire field when defining our LIM. Together these 15 explain about $85 \%$ of the total variance. Just as Alexander et al. (2008) and Newman (2007) found when constructing LIMs for observed Pacific SST, we find the resulting LIM is appropriate for North Pacific subsurface temperature variability in CCSM3 and approximates its behavior well. One indication of this is that when we generate our LIM with $\tau_{0}=1$ year, the resulting propagator Eq. 6 explains $66 \%$ of yearly increments and $41 \%$ of 2 year increments while the corresponding values for persistence forecasts are $49 \%$ and $-6 \%$, respectively. Moreover, other conditions that a LIM should meet (Penland and Sardeshmukh 1995) if the assumptions it is based on are valid are also satisfied. The Euclidean norm of $\mathbf{B}$ is insensitive to variation of $\tau_{0}$ in the 1-3 year range, and the eigenvalues of $\mathbf{Q}$ are all positive. Additional indications of the appropriateness of the LIM are presented in Sect. 5 where we find it can reproduce the basic predictability characteristics of the three CCSM3 ensemble experiments of our study.

\section{Prominent modes}

To identify prominent modes of subsurface variability that are intrinsic to CCSM3, we apply EOF analysis to the North Pacific in the control integration. Any one method of identifying modes is not necessarily adequate for all situations, but as seen below, EOFs are effective at identifying the mode of North Pacific variability that we are most interested in, the PDO. When we calculate EOFs for the average temperature in the upper $300 \mathrm{~m}$, we find the first two EOFs together explain about $40 \%$ of the total variance, and they are well separated from the other EOFs (North et al. 1982). We mainly focus on the characteristics of these two EOFs. For comparison, we also examine the first two EOFs of SST in the same domain because this is the variable on which many studies have concentrated.

\subsection{The first two EOFs}

SST EOF1 (Fig. 2, top left) has a spatial pattern similar to observations (Mantua et al. 1997) and to that in an earlier version of CCSM (Kwon and Deser 2007). There are anomalies of one sign in the western and central North Pacific along the Kuroshio Extension at around $40^{\circ} \mathrm{N}$, surrounded by anomalies of the opposite sign. EOF1 in upper $300 \mathrm{~m}$ temperature (Fig. 2, bottom left) generally looks like the SST EOF1, except that the two active centers at the Kuroshio extension and the Central Pacific are better separated in the subsurface. Power spectra (Fig. 3) of the first principal component (PC) in SST and subsurface temperature are qualitatively similar in that both are distinctly red with multiple apparent peaks for periods longer than about 10 years. The dominant SST frequency is slightly higher than in control runs of CCSM2 (Kwon and Deser 2007) and in a T31 version of CCSM3 (Zhong et al. 2008); the latter two experiments have dominant peaks at 16 and 15 years, respectively. When we examine the corresponding spectra based on only the first half and only the second half of the dataset we find that, except for the SST peak at a period of
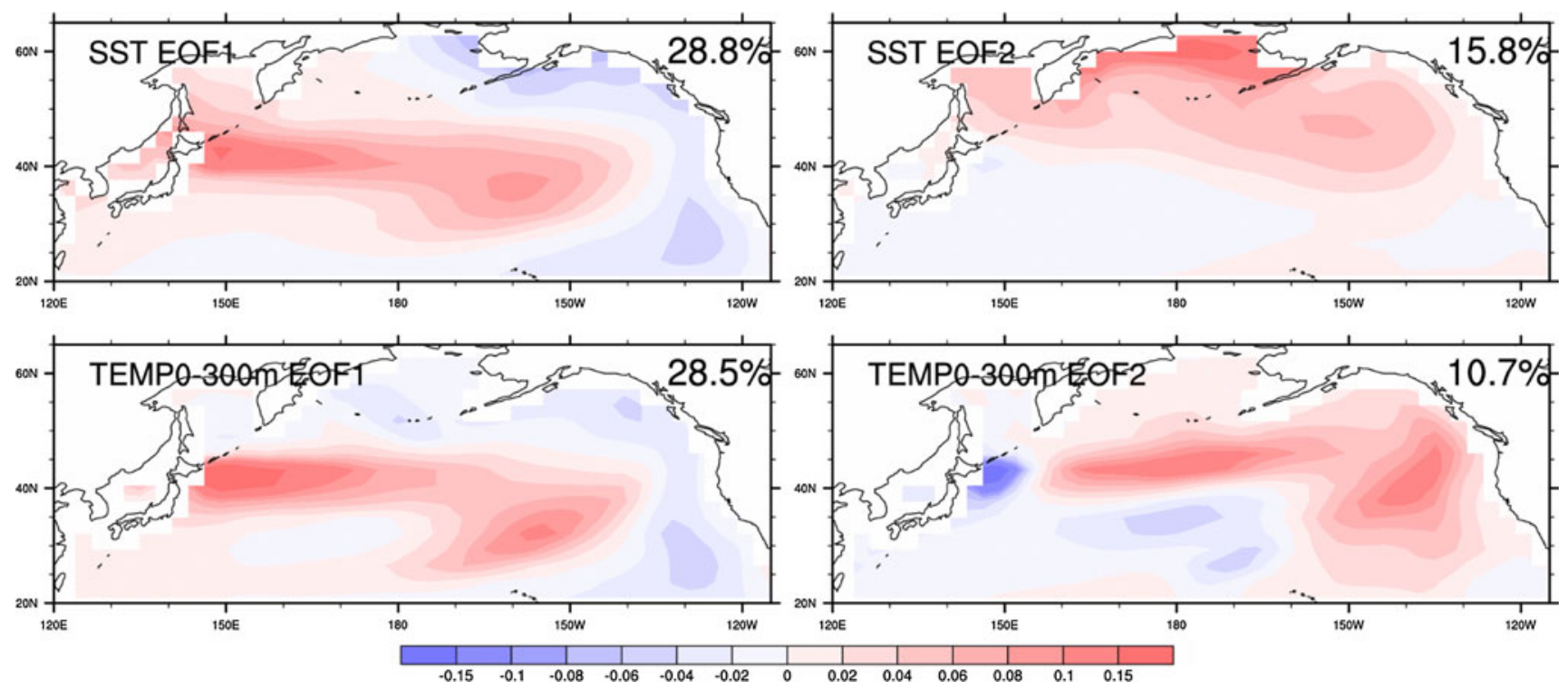

Fig. 2 EOF1 (left) and EOF2 (right) of annual mean SST (top) and upper-300 m temperature (bottom) in the North Pacific $\left(120^{\circ} \mathrm{E}-110^{\circ} \mathrm{W}\right.$, $20^{\circ} \mathrm{N}-65^{\circ} \mathrm{N}$ ) from the 700 year control run. The percent interannual variances explained are given in each panel 

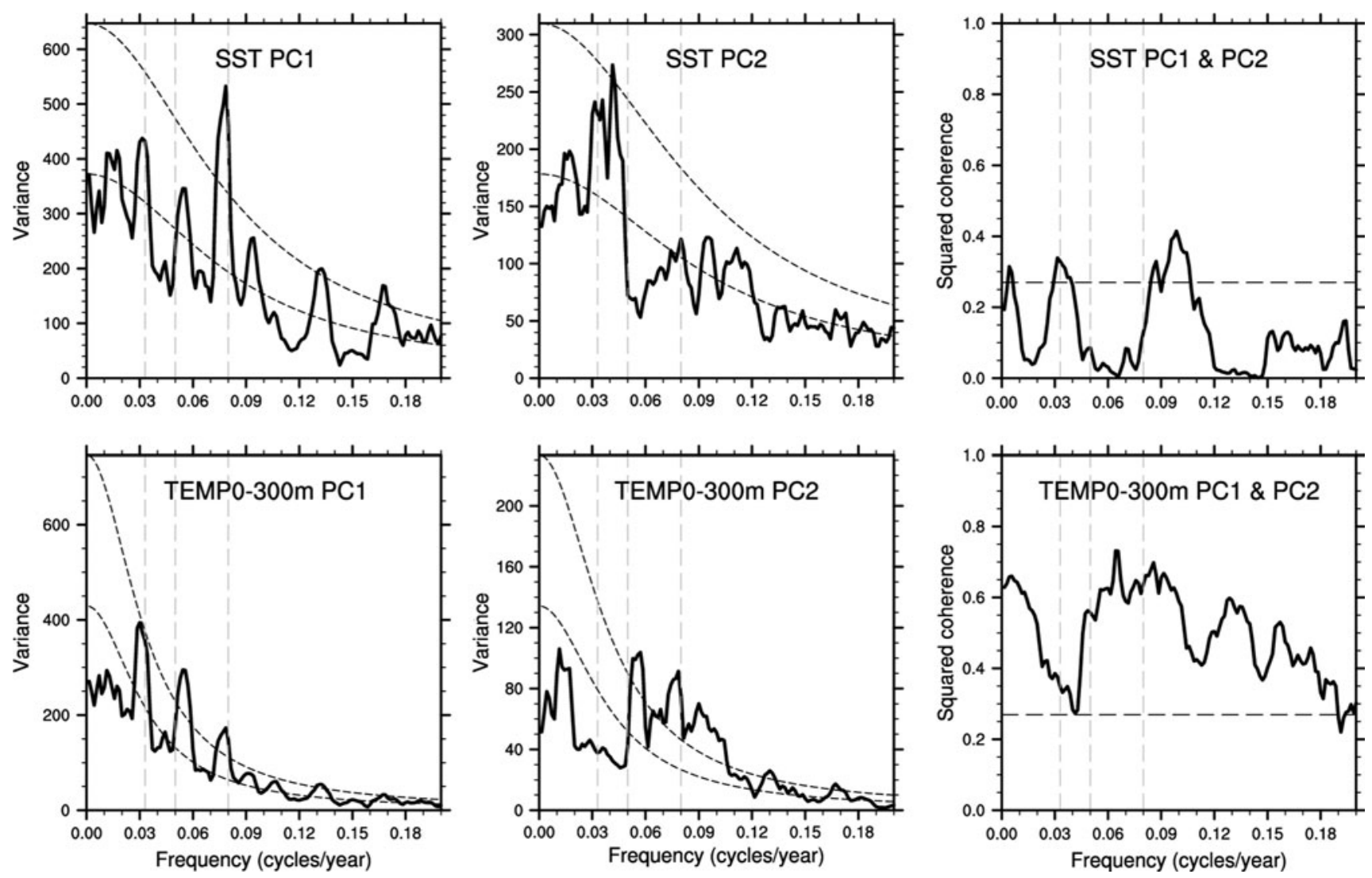

Fig. 3 Power spectra for principal component (PC) of SST EOF1 (top left), SST EOF2 (top middle), upper-300 m temperature EOF1 (bottom left), and upper-300 m temperature EOF2 (bottom middle) in the control run. The two black dashed lines are first-order autoregressive model (AR1) spectra and 99\% significance level based on the 1 year lag autocorrelations of the time series. The right panels are the

12 years, the exact placement of these peaks is not robust. We do find, however, that there is a preference for unusually strong peaks in the subsurface in the 10-30 year range and that the subsurface spectrum has a sharper drop-off as frequency increases than the SST spectrum.

In contrast to EOF1, EOF2 is very different for SST and subsurface temperature (Fig. 2, right panels). The SST pattern is concentrated in high latitudes while the subsurface pattern consists of features in midlatitudes, including two lobes that resemble eastward shifts of features in EOF1. The subsurface temperature PC2 has wide frequency peaks ranging from 10 to 20 year periods (Fig. 3, bottom middle) but only the peak at 12 years is present in both dataset halves. PC2 for SST has no distinct peaks in this range.

To determine whether the EOF patterns may be two phases of a time evolving feature, we carry out a coherency analysis. While the leading two PCs of SST are related at only the 30 and 10 year periods (Fig. 3, right panels), the two PCs of subsurface temperature have significant coherency at almost all frequency bands, and the coherency coherence between the leading two PCs in SST (top) and upper$300 \mathrm{~m}$ temperature (bottom) and the dashed line indicates the $99 \%$ significance level. The three grey vertical reference lines denote frequencies corresponding to 12,20 and 30 year periods. The spectra are based on 7-point modified Daniell smoothing

is much stronger than that between the two PCs in SST. The tight relationship between the two PCs in the subsurface temperature suggests that they correspond to a time dependent physical mode. Taking into consideration their power spectra, this physical pair has a preference for variability in the 10-30 year range.

\subsection{Leading propagating mode}

If there is a prominent time evolving mode in the subsurface temperature whose variability is approximately sinusoidal, complex EOF (CEOF) analysis (von Storch and Zwiers 1999) should capture it. When we apply CEOF analysis to 10-30 year filtered subsurface temperature in the North Pacific, we find the real and imaginary parts of CEOF1 resemble EOF1 and EOF2, respectively (Fig. 4 bottom panels). They explain $47 \%$ of the variance in this band. This is consistent with the possibility that subsurface temperature EOF1 and EOF2 are linked. Furthermore, since implicit in the CEOF analysis is that the imaginary part of a CEOF tends to follow the real part, it is apparent 

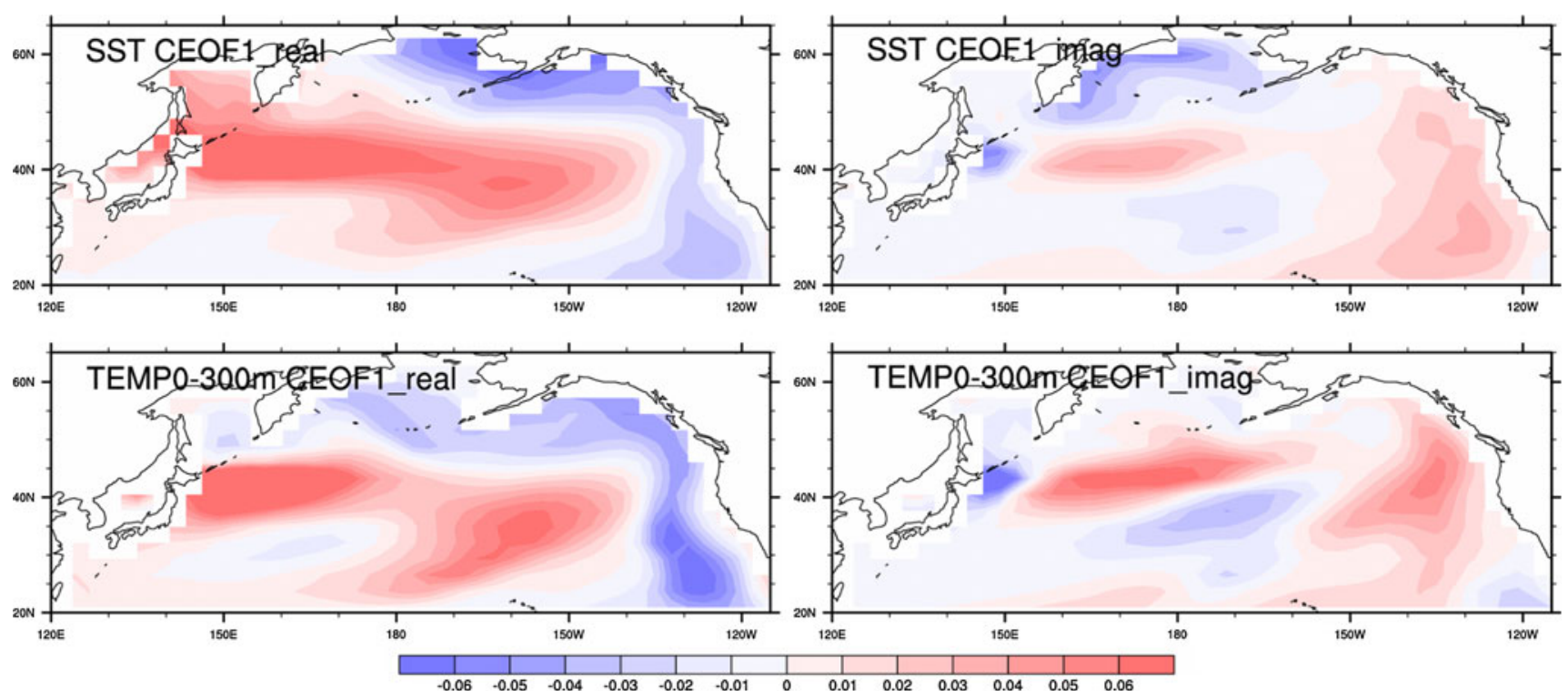

Fig. 4 Real (left) and imaginary (right) part of complex EOF1 of 1030 year filtered SST (top) and upper-300 m temperature (bottom) in the North Pacific in the control run. CEOF1 explains $42.4 \%$ and

$47.4 \%$ of total variance of 10-30 year filtered SST and upper-300 m temperature, respectively

from Fig. 4 that the mode that CEOF1 represents is an eastward propagating mode.

We also apply CEOF analysis to band-pass filtered SST to detect the dominant propagating mode at the surface. Interestingly, both the real and imaginary parts of SST CEOF1 are similar to the corresponding components of CEOF1 in the subsurface temperature, suggesting that there are consistent changes in SST associated with the leading propagating mode in the subsurface temperature. In agreement with this interpretation, projections of bandpass filtered fields onto the real part of subsurface CEOF1 and onto the real part of SST CEOF1 have a correlation coefficient of 0.57 as do projections onto the imaginary parts of the CEOFs. The real part of SST CEOF1 also looks like SST EOF1, but the imaginary part is completely different from the SST EOF2, indicating that the leading propagating mode is more prominent in the subsurface temperature than at the surface. In fact, we find in results not shown, that the imaginary part of SST CEOF1 projects most strongly onto the fifth EOF of SST, a pattern that represents only about $4 \%$ of SST variability. Hereafter, we focus on the prominent modes in the subsurface temperature only, but we will examine whether their expression in SST, as suggested by the above results, is significant. Since the first two subsurface EOFs are similar in structure to CEOF1, for the sake of simplicity, we use the leading two EOFs to define the leading propagating mode in the remainder of our study.

Although CEOF1 represents the strongest signature that can be approximated by an oscillation in the dataset, the analysis can still correspond to a pair of patterns that are not a true oscillation. To investigate whether there is true periodicity in the variability of the patterns that make up CEOF1, we employ auto- and lag-correlations of PC1 and PC2 (Fig. 5). The PC1 autocorrelation first crosses zero in year 8 (Fig. 5a). Thereafter, it remains close to zero, and there is no indication of growth of the opposite phase. The PC2 autocorrelation first drops below zero in year 7 (Fig. 5d). Although PC2 seems more oscillatory than PC1, the autocorrelations are weak after the first zero-crossing. The strongest lag correlation is found when PC2 lags PC1 (Fig. 5b). There are also significant lag correlations when PC1 lags PC2 (Fig. 5c), but the amplitude is much smaller than when PC1 leads PC2. Taken together, these results indicate that PC2 tends to follow PC1 but PC1 only weakly follows PC2. Physically, this means that the pair of midlatitude temperature anomalies at $150 \mathrm{E}$ and $160 \mathrm{~W}$ in EOF1 tends to propagate eastward leading to the lobes at $170^{\circ} \mathrm{E}$ and $140^{\circ} \mathrm{W}$ in EOF2. But the features in EOF2 have only a weak tendency to evolve to (minus) the features in EOF1. Therefore, we prefer to call the first two EOFs the leading propagating mode rather than the leading oscillation.

\subsection{Composite and heat budget analysis}

To give a more complete representation of the evolution of the leading propagating mode we use composites applied to 10-30 year bandpass fields from the control. We define the phase of the propagation by $\arctan \left(\frac{p c 2^{\#}}{p c 1^{\#}}\right)$, where \# means the PCs are normalized by their standard deviation. Subsurface temperature composites at phase $0^{\circ}, 45^{\circ}, 90^{\circ}$, and 

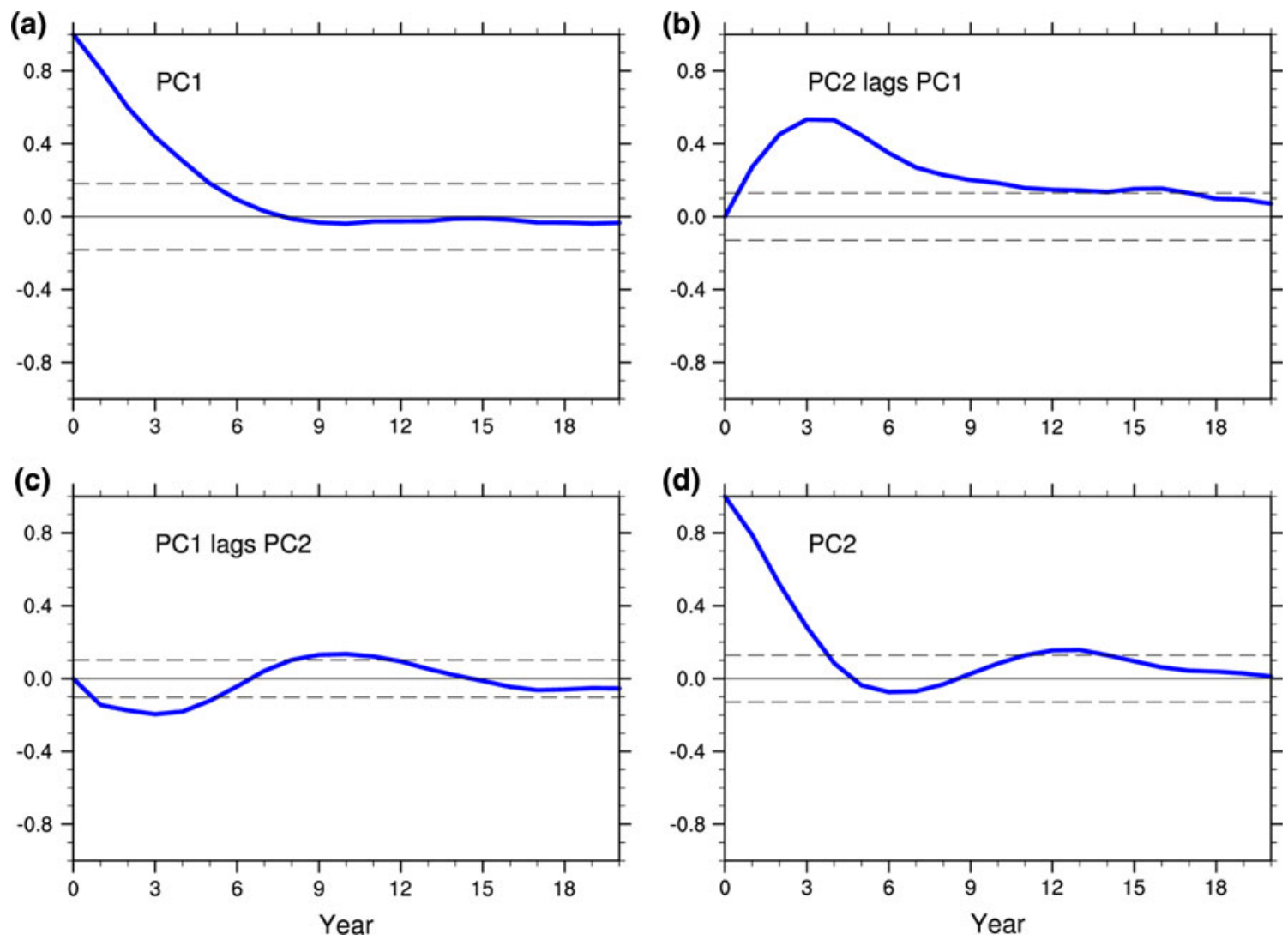

Fig. 5 a and $\mathbf{d}$ are autocorrelations of PC1 and PC2 of the upper-300 m temperature in the 700 year control run, and $\mathbf{b}$ and $\mathbf{c}$ are lag correlations between PC1 and PC2. The grey dashed lines indicate the 99\% significant level

$135^{\circ}$, with about 60 states contributing to each, are shown in the left panels of Fig. 6. As expected the composites correspond to the eastward propagation of the two midlatitude warm anomalies present at phase $0^{\circ}$ until they reach the northern and eastern boundary of the basin at phase $135^{\circ}$. Meanwhile, negative anomalies appear off the coast of Japan and in the central Pacific at phase $90^{\circ}$, and strengthen at phase $135^{\circ}$.

Using the phase of the subsurface temperature, we also generate composites of SST and sea level pressure (SLP) (Fig. 6, right panels). The structure of the SST anomalies agrees with the subsurface temperature anomalies at all four phases, confirming that the leading propagating mode in the subsurface temperature is associated with consistent temperature anomalies at the surface. Interestingly, there are systematic changes in SLP as well. Positive SLP anomalies prevail in the North Pacific at phase $0^{\circ}$. Thereafter, the high pressure center moves slightly northwestward and negative anomalies appear in midlatitudes and propagate slowly northward. A previous study has found evidence for two-way coupling between SST and the atmospheric circulation on the decadal time scale in CCSM2 (Kwon and Deser 2007). Here we do not attempt to establish cause and effect but rather simply point out there is a linkage between the subsurface propagating mode and SLP. Although the amplitude of the SLP anomalies is relatively weak (about $0.5 \mathrm{hPa}$ at the center) compared to interannual variability $(\sim 3 \mathrm{hPa})$, the persistence of the SLP anomalies may cause a significant impact on other surface variables that are of direct relevance to humanity.

Next we try to understand what processes produce the propagation, as the answer may help assess the prospects for decadal predictability of the propagating mode. To accomplish this, we apply the temperature tendency equation to depth averaged conditions in the upper $300 \mathrm{~m}$,

$\frac{\partial T}{\partial t}=-\frac{Q_{\text {net }}}{\rho_{0} C_{p} H}+A+B+$ residual

where $T$ is depth averaged temperature, $Q_{n e t}$ is the net airsea surface heat flux, $H$ is the layer thickness $(=300 \mathrm{~m})$, $\rho_{0}$ is depth averaged density, $C_{p}$ is heat capacity, $A$ and $B$ are horizontal and vertical convergence of temperature flux, respectively, averaged over $300 \mathrm{~m}$. Note that we replace the temperature advection term in the conventional temperature tendency equation with temperature flux convergence because temperature flux is a standard output of the model, and such a change is valid under the assumption that the three dimensional divergence equals zero. We first 

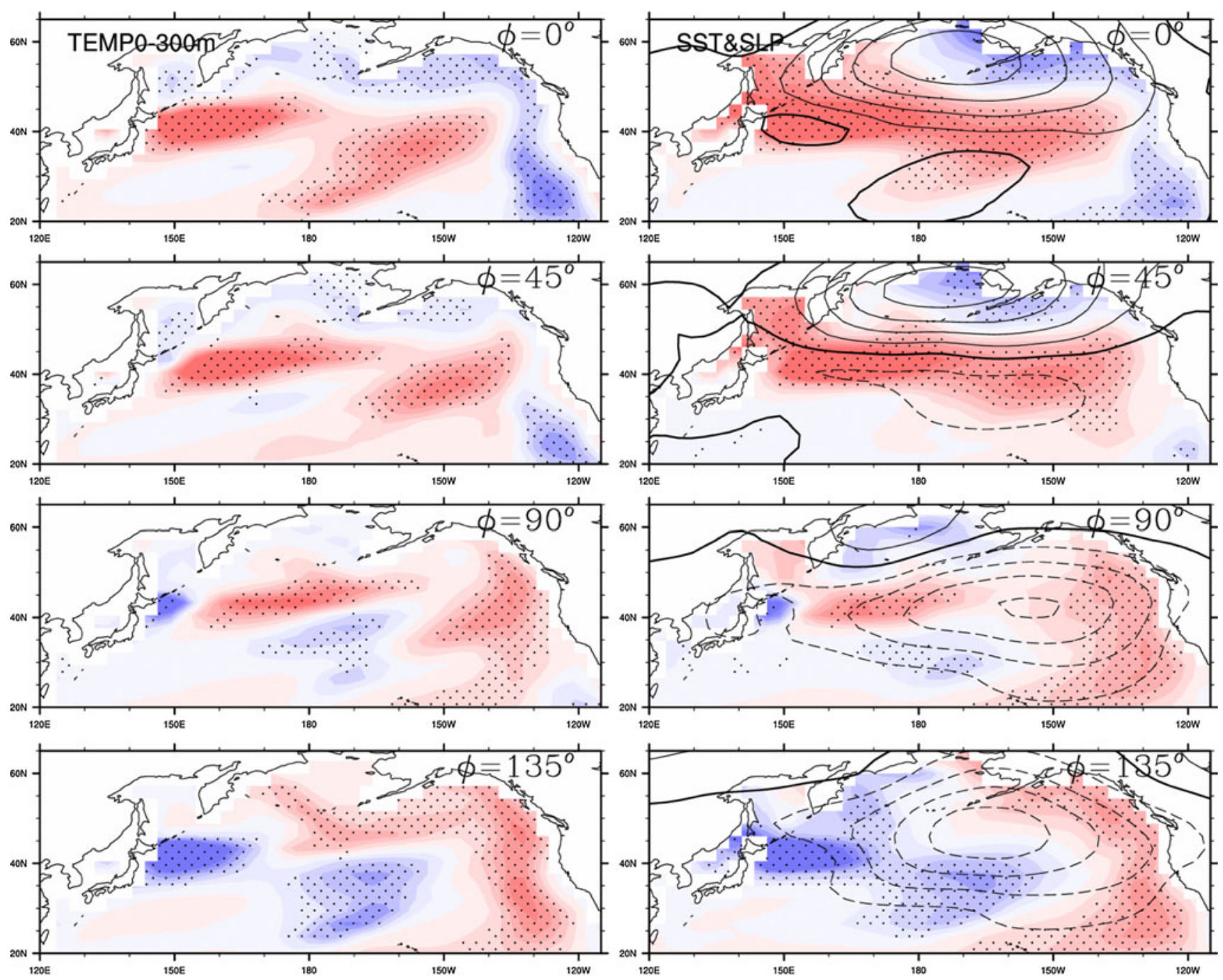

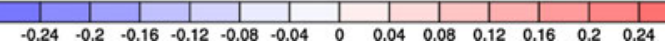

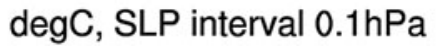

Fig. 6 Composite of 10-30 year bandpass filtered upper-300 m temperature (left panels), SST (shading, right panels) and SLP (contour, right panels) at phase $0^{\circ}, 45^{\circ}, 90^{\circ}$ and $135^{\circ}$ of the subsurface propagating mode with the phase calculated using

calculate the horizontal convergence of temperature flux on the native grids at each layer, followed by the vertical averaging. As for $\mathrm{B}$, in this equation for depth average temperature, it equals the vertical temperature flux at $300 \mathrm{~m}$. Other terms including diffusion and penetration of solar radiation etc. make up the residual. Note the relationship in Eq. 8 remains valid if we bandpass filter each term provided we make clear that the filtering is of each term, not of each variable. Below we apply Eq. 8 with each term filtered by the same 10-30 year filter used in the Fig. 6 composites. We denote this filter by a prime.

In Sect. 3b, we have constructed $T^{\prime}$ composites at several different phases (Fig. 6, left panels). The subsurface temperature change from phase $0^{\circ}$ to $90^{\circ}$ is plotted in Fig. $7 \mathrm{~d}$. normalized PC1 and PC2 of upper-300 m temperature in the control run. Each composite map is derived from about 60 episodes and the stippling indicates the $95 \%$ significance level of Student's $t$ test

Now we want to quantify how much each of the three terms on the right-hand side of Eq. 8 contributes to this temperature change. To do this, we make composites of the three terms, $-\frac{Q_{\text {net }}^{\prime}}{\rho_{0} C_{p} H}, A^{\prime}$, and $B^{\prime}$ at phases $15^{\circ}, 45^{\circ}, 75^{\circ}$. Assuming it takes about 1 year for the leading propagating mode to develop $30^{\circ}$ (consistent with the robust peak at 12 years we saw in several Fig. 3 spectra), we multiply the composite of the three terms at phases $15^{\circ}, 45^{\circ}, 75^{\circ}$, respectively, by 1 year to get the corresponding $\Delta T^{\prime}$ from phase $0^{\circ}$ to phase $30^{\circ}$, from $30^{\circ}$ to $60^{\circ}$, and from $60^{\circ}$ to $90^{\circ}$. Summing $\Delta T^{\prime}$ from each phase for each term in the tendency equation yields the contribution to the total temperature change in Fig. $7 \mathrm{~d}$ by the surface heat flux 
(Fig. 7a), horizontal convergence of temperature flux (Fig. 7c) and vertical temperature flux at the layer bottom (Fig. 7e). If we add the three contributions arrived at in this fashion (Fig. 7b) we see that this procedure does produce tendencies which together correspond to the composite temperature change both in terms of structure and amplitude.

Examining the contributions from the three terms we find the horizontal convergence of temperature flux (Fig. 7c) plays the dominant role. We further decompose this term into horizontal temperature advection $-\left(u \frac{\partial T}{\partial x}+v \frac{\partial T}{\partial y}\right)$ and the horizontal convergence term $-T\left(\frac{\partial u}{\partial x}+\frac{\partial v}{\partial y}\right)$. Again these are calculated for each model layer and then depth averaged. We find both terms are important to horizontal convergence of temperature flux
(Fig. 7b) over the Kuroshio Extension region (figure not shown). In most of the North Pacific basin away from this region, horizontal advection dominates (figure not show). To determine whether advection by the mean currents is primarily responsible for this advection, we recalculate the horizontal advection term with the currents replaced by their climatological mean value (Fig. 7f). Though this component of advection is important, major features in Fig. 7c, particularly in the Kuroshio Extension region, are not reproduced by this simplification. This implies that perturbation currents cannot be ignored and raises the possibility that atmospheric anomalies are involved in producing the eastward propagation of the mode.

Unlike horizontal convergence of temperature flux, the temperature flux at $300 \mathrm{~m}$ depth (Fig. 7e) has the opposite sign of the total temperature change (Fig. 7d) in most (a) surface heat flux

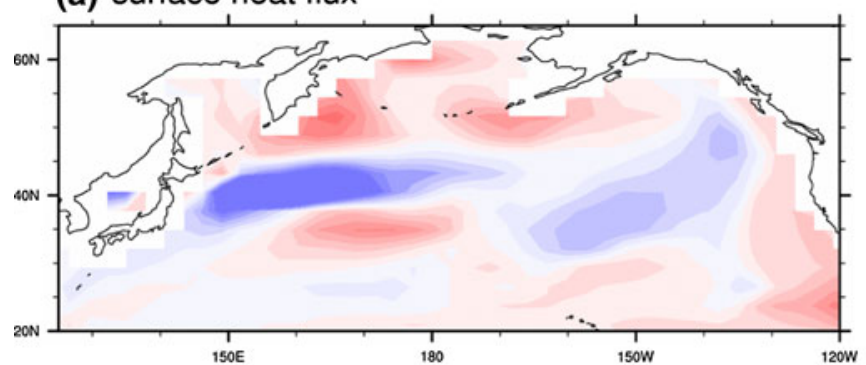

(c) horizontal convergence of temp flux

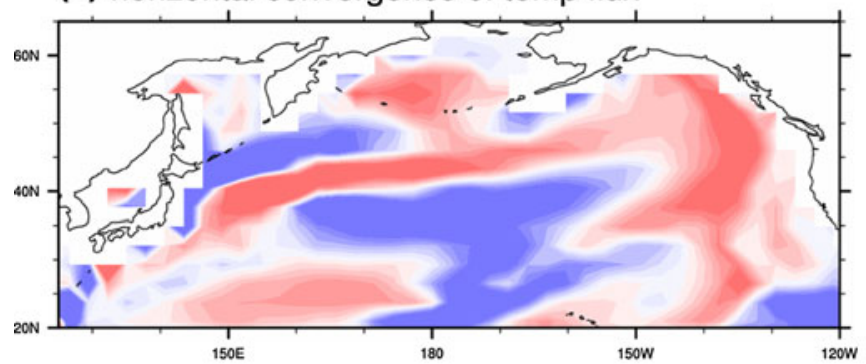

(e) vertical temp flux @300m (b) a)+c)+e)

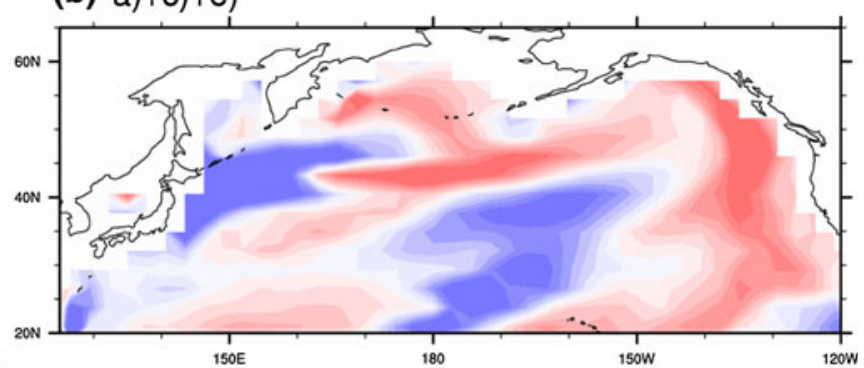

(d) $\mathrm{T}^{\prime}\left(\phi=90^{\circ}\right)-\mathrm{T}^{\prime}\left(\phi=0^{\circ}\right)$

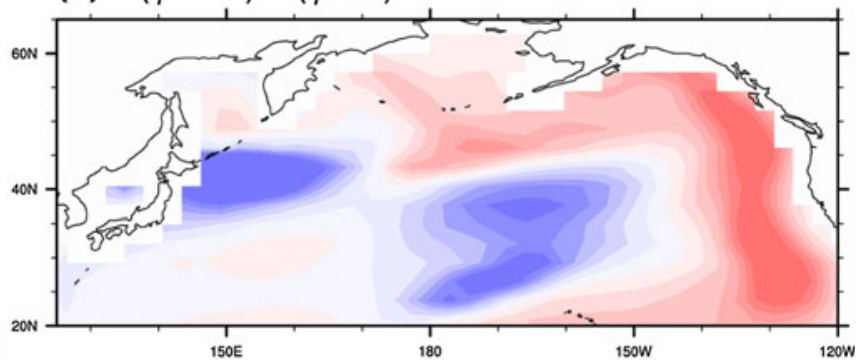

(f) climatological $\mathrm{u}, \mathrm{v}$ advect $\mathrm{T}$ '

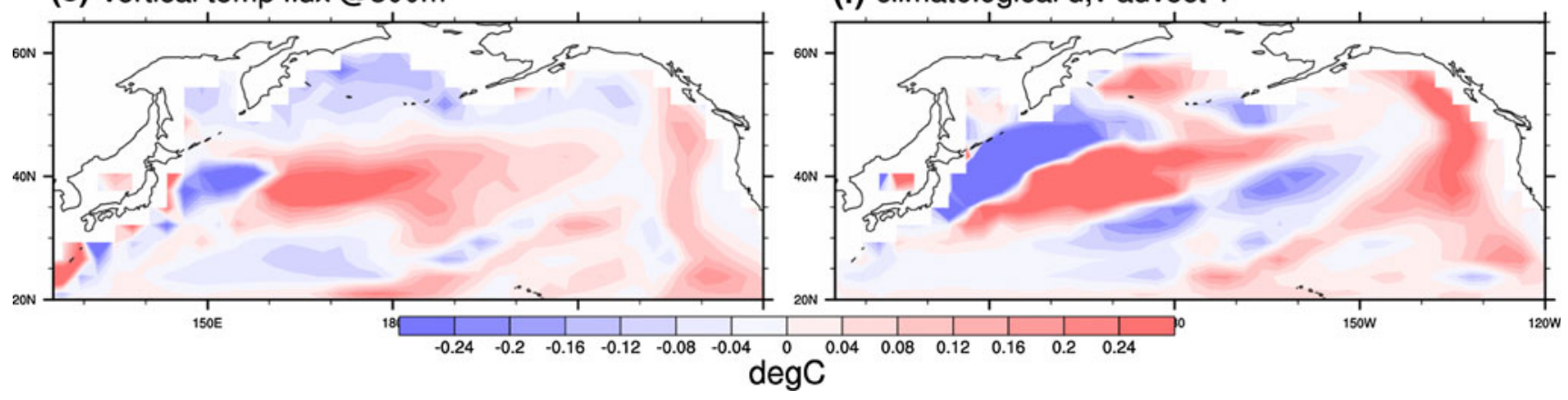

Fig. 7 Contributions from 10-30 year filtered net surface heat flux (a), horizontal convergence of temperature flux (c) and vertical temperature flux at $300 \mathrm{~m}$ depth (e) to 10-30 year upper-300 m temperature change (d) from phase $0^{\circ}$ to phase $90^{\circ}$ of the subsurface propagating mode. All panels are composite maps based on about 60 episodes from the control run (method described in Sect. 3c) (b) is the sum of panels (a), (c) and (e), and (f) represents the temperature change from phase $0^{\circ}$ to phase $90^{\circ}$ due to climatological mean currents advecting 10-30 year filtered temperature anomalies, which is one component of (c) 
regions, except at the beginning of the Kuroshio Extension and in the eastern Pacific. Therefore, it is not the main cause for the eastward propagation. The net surface heat flux (Fig. 7a) does contribute positively to the eastward propagation, especially in the Kuroshio Extension, but overall, the eastward propagation is largely driven by horizontal advection. We also calculate the composite for the residual in Eq. 8, and it explains the difference between Fig. $7 d$, b. It is smaller than the contributions from surface heat flux and the advection terms. Given the fact that the analysis does not rule out the possibility that atmospheric processes, with their high variability, are important contributors to the evolution of the propagating mode associated with the model PDO, prospects for it having long predictability are diminished.

\section{Predictability of the leading EOF modes in the three ensembles}

Having found nearly $40 \%$ of the interannual subsurface variability is composed of a pair of patterns that act together, next we examine initial-value predictability of this pair in the three ensembles. This involves examining the PCs resulting from projecting the intrinsic component of temperature (cf. sect. 2) onto control run EOF1 and EOF2. Despite the common perception that ensemble spread is the main determinant of predictability, we find both the mean and spread make significant contributions.
Ensemble means of PC1 and PC2 as a function of prediction time are presented in Fig. 8a, b. As mentioned earlier, each ensemble has unique characteristics in its initial condition. The initial values of PC1 and PC2 in Ensemble I corresponds to the 68th and 66th percentile of the control amplitude, respectively. Since the one standard deviation boundaries of a normal distribution equal the 16th and 85th percentiles, Ensemble I's initial values for PC1 and PC2 are both small. Ensemble II has an extremely large positive PC1 (99th percentile) but a small negative PC2 (37th percentile), and Ensemble III has a small positive PC1 (70th percentile) but a very large positive PC2 (92nd percentile). In the first 20 years of integration, the mean PCs of Ensemble I oscillate but the small amplitudes do not give much predictive information. In Ensemble II as PC1 decays PC2 strengthens in the first 6 years. Ensemble III also starts with strong amplitude in a leading PC, but in this case it is PC2 and as that coefficient decays there is no compensation in PC1. The difference between Ensemble II and III is consistent with our analysis of intrinsic variability in the previous section where we found that a strong EOF1 pattern can lead to a strong EOF2 pattern, but a strong EOF2 does not necessarily produce a strong negative EOF1.

As for the ensemble spread (Fig. 8c, d), in all three ensembles during the first 8 years the standard deviation is less for normalized PC2 than for normalized PC1; it takes about 5 years for the standard deviation of PC1, and 8 years for that of PC2, to exceed one standard deviation of
Fig. 8 40-member ensemble mean subsurface temperature PC1 (top left) and PC2 (top right), and normalized ensemble standard deviations of PC1 (bottom left) and PC2 (bottom right) in the three ensembles. The PCs are calculated by projecting the detrended upper$300 \mathrm{~m}$ temperature in the North Pacific onto the corresponding EOFs from the control run
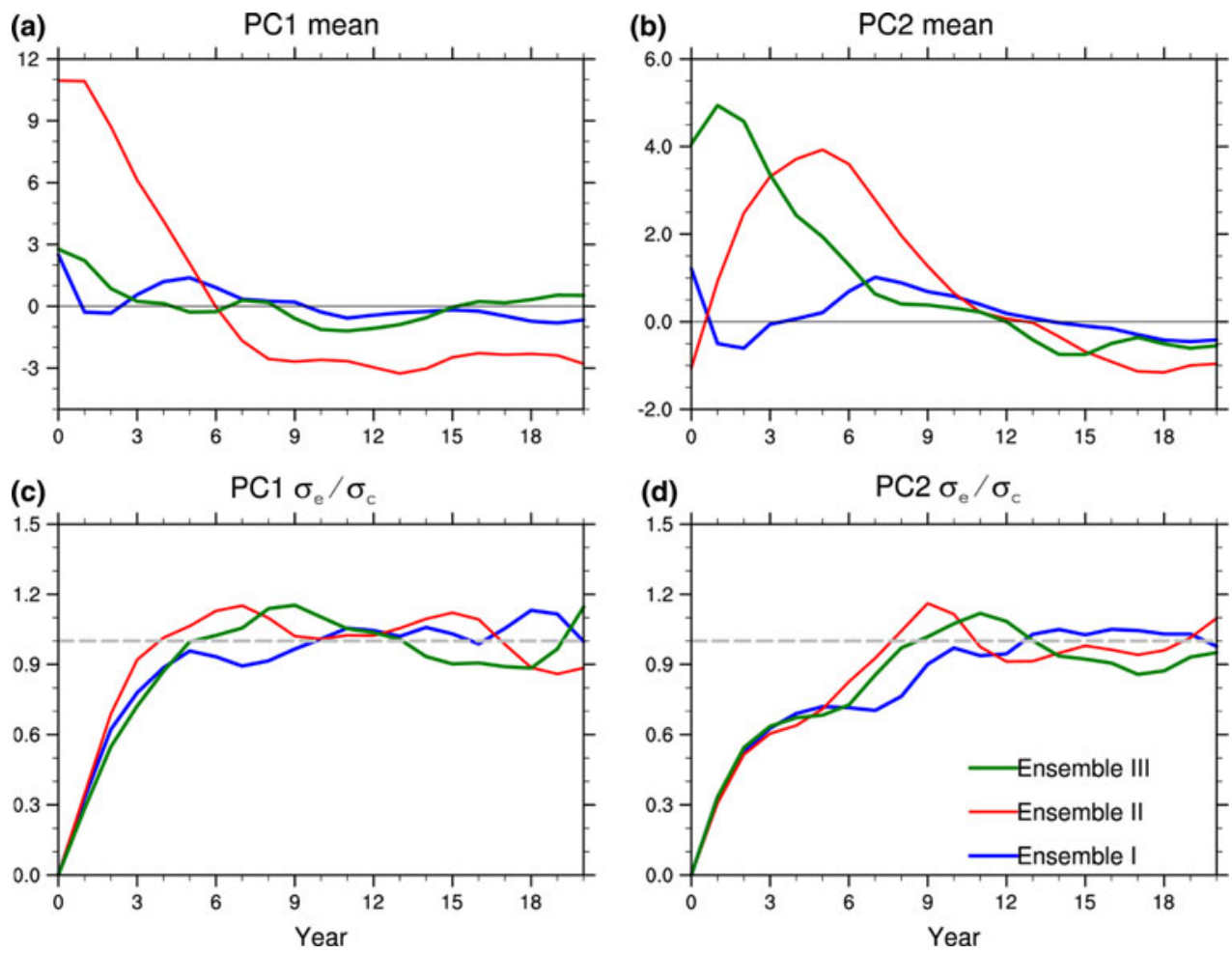
the control run. So from the standpoint of spread, predictability of PC2 is lost much later than for PC1. Another interesting feature is that the spread in all three ensembles grows at a similar pace, despite the large differences among the initial conditions. This latter fact will be important in the next section. When considering Fig. 8, it is important to recognize that the range at which the standard deviation of an ensemble exceeds the climatological standard deviation is only a rough indication of when predictability is lost. For an infinite ensemble the approach is asymptotic and so the predicted standard deviation is never greater than the climatological standard deviation, while for a finite ensemble sampling effects can erroneously produce an apparent convergence of the two distributions before it has actually taken place.

As we have pointed out, relative entropy measures the difference between predicted and climatological distributions in terms of both mean and spread. We use univariate relative entropy of PC1 and PC2 (Fig. 9 left and middle panels), and bivariate relative entropy of the two PCs (Fig. 9 right panel) to quantify the predictability of EOF1 and EOF2 and of the leading propagating mode, respectively. Furthermore, we decompose the relative entropy into its signal and dispersion components to distinguish the effects of departures of the mean and spread from climatology. The grey dashed lines in Fig. 9 show the 95\% significance values for 40-member ensembles as derived in the way explained in Sect. 2. We consider predictability to be lost when relative entropy dips below this level.

In both Ensemble I and III, where the initial projection onto PC1 is small, $\mathrm{PC} 1$ is predictable for about 4 years, and the predictability mainly comes from the dispersion component (Fig. 9, left column). PC1 is more predictable in Ensemble II due to large contributions from the signal component (Fig. 9, left middle). The total relative entropy does not drop to the threshold level until year 6 when the
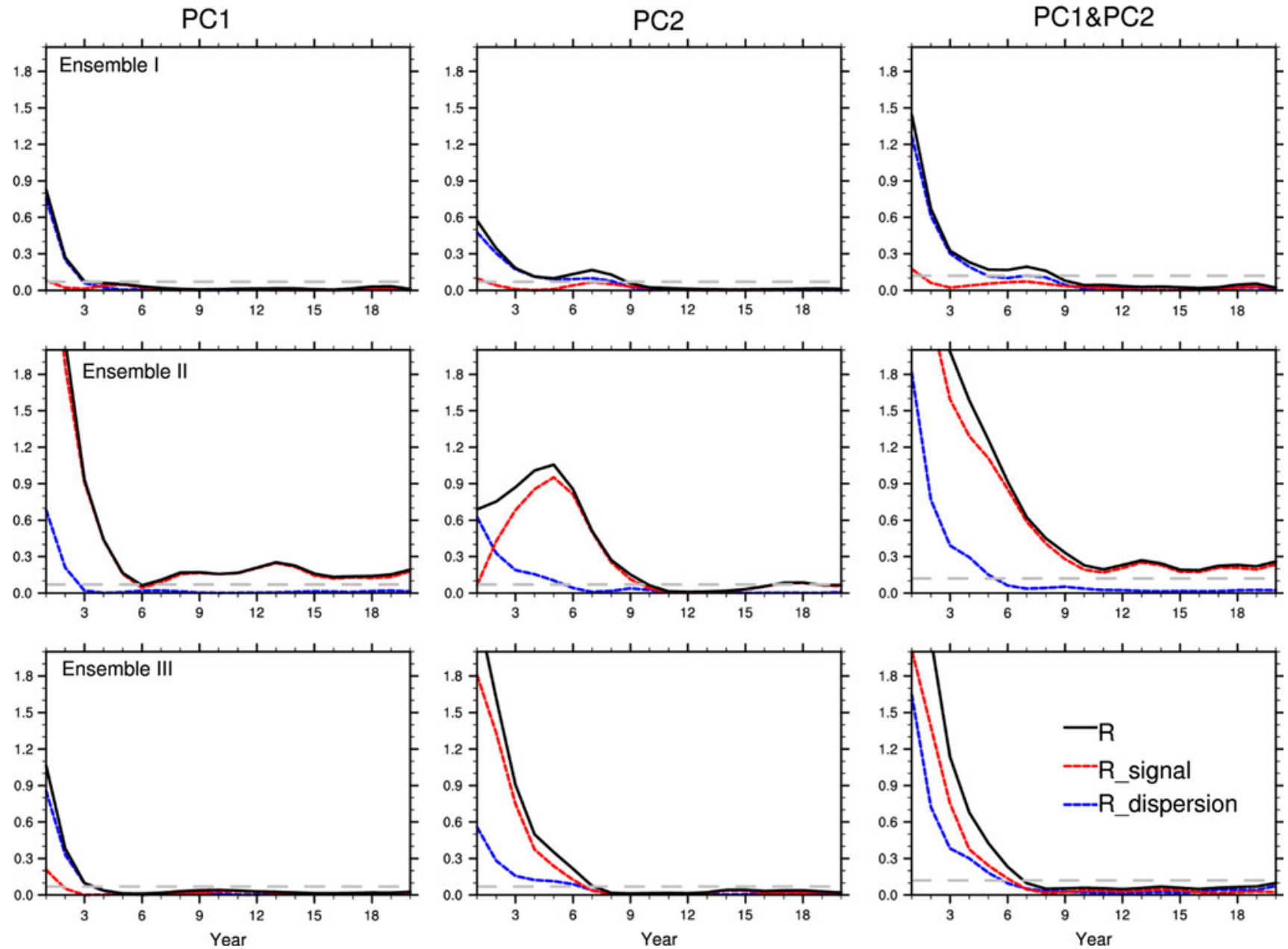

Fig. 9 Univariate relative entropy for PC1 (left) and PC2 (middle) and bivariate relative entropy for PC1 and PC2 (right) of the upper$300 \mathrm{~m}$ temperature. Top, middle, and bottom panels represent Ensemble I, II, and III, respectively. The grey dashed lines indicate

the $95 \%$ percentile of relative entropy values from 40 random states (drawn 10,000 times) in the control run. The black, red, and blue lines indicate the total relative entropy, its signal component, and its dispersion component, respectively 
ensemble mean drops to zero. From year 6 to year 20, relative entropy stays above the threshold as the mean PC1 remains different from climatology.

For all three ensembles, $\mathrm{PC} 2$ is more predictable than PC1 and the enhanced predictability comes from two sources. First, the dispersion component of relative entropy for PC2 decays more slowly than for PC1, which is consistent with Fig. 8c, d. Second, when the prediction starts with a strong PC1, the initial signal propagates to PC2 (as in Ensemble II), but a strong initial PC2 signal does not always produce a strong PC1 (as in Ensemble III). The combination of these two factors is that $\mathrm{PC} 2$ is predictable for 7-10 years in our experiments (Fig. 9, middle panel).

We have argued that EOF1 and EOF2 are dynamically linked through advection, so it makes sense to consider the predictability of PC1 and PC2 simultaneously. This is done in the right column of Fig. 9, which displays bivariate relative entropy for these two quantities. Again we see predictability in the 7-10 year range. Ensemble I benefits from contributions from both PCs. Ensemble II has the longest predictability with our predictability criterion even being met at year 20, but keep in mind that the strong initial projection onto EOF1 that is probably responsible is a very rare (99th percentile) event. Ensemble III is not especially predictable in spite of a strong initial anomaly, presumably because PC2 tends to simply decay. We also note that though the initial projections onto EOF1 and EOF2 are weak in Ensemble I, they stay predictable for approximately as long in this experiment as in Ensemble III. This suggests that other system components can influence the evolution of these structures.

\section{Predictability estimated from a linear stochastic model}

As explained in Sect. 2, we have constructed a LIM that approximates CCSM3. In that section we explained why it is reasonable to think that our LIM behavior should be a good approximation to CCSM3 subsurface temperature behavior. Further evidence of its validity is the fact that we found in Sect. 4 that there is no obvious dependence of the rate at which PCs disperse on the particular initial conditions employed in our three ensembles. Recall from Sect. 2 that this is a property that one expects to be true for systems that satisfy the assumptions that LIMs are based on. As a final test of the appropriateness of the LIM for our predictability investigation, we evaluate whether it can reproduce major predictability characteristics of the first two PCs seen in the three CCSM3 ensemble experiments.

For each of the three ensemble experiments we use the year 0 values of the first 15 PCs as an initial condition and use Eq. 6 and Eq. 7 to estimate the mean and standard deviation of the distributions that would occur each year in (infinite) ensembles of forecasts. These are compared with values from the corresponding CCSM3 ensemble in Fig. 10. For both PCs, the LIM reproduces the mean in the first 6 years in each ensemble (Fig. 10a, b) including the propagation of the signal from the PC1 to PC2 in Ensemble II. Moreover, it reproduces Ensemble IIIs characteristic of having a strong PC2 that does not initiate later growth in PC1. The spread of the LIM ensembles is also a good match to CCSM3 behavior (Fig. 10c, d). The LIM result saturates at a similar rate as the spread in each of the three ensembles thus reproducing the tendency of PC2 spread to saturate more slowly than that of PC1. The similarity of the evolution of CCSM3 and LIM ensembles indicates the LIM is indeed an appropriate tool for investigating predictability properties of the leading CCSM3 modes.

Now we use the LIM to see whether the predictability properties of $\mathrm{PC} 1$ and $\mathrm{PC} 2$ that are present in the three CCSM3 ensembles are valid for other initial states. For it may be that attributes other than the initial values of PC1 and PC2 have influenced the characteristics we have noted. First, to consider cases with strong PC1, we select the 50 states from the 700 year control run with the strongest PC1, and use them as initial conditions for 50 LIM solutions, again employing Eq. 6 and Eq. 7. For each initial condition, we calculate the relative entropy of $\mathrm{PC} 1$ and $\mathrm{PC} 2$, individually and combined. The 50 case average relative entropy and its signal and dispersion components, as well as the envelope of relative entropy for all 50 cases, are presented in the top panel of Fig. 11. These results suggest that two of the major attributes we saw in the CCSM3 experiments are likely to be more generally valid: first, predictability in strong PC1 amplitude cases is dominated by contributions from the signal component; second, signals propagate from EOF1 to EOF2, and EOF2 can extend the lifetime of information initially in EOF1. Note from the envelope of solutions in the figure that this second attribute is not true for all initial conditions, but it does hold for most situations. ${ }^{1}$

Next, we consider cases with strong PC2 initial conditions. We select the 50 states from the control run with the strongest PC2, and repeat the above LIM simulation and analysis procedures. When we do this we confirm our main result concerning this pattern, namely that there is little if any tendency for initial information in $\mathrm{PC} 2$ to transfer to PC1 (Fig. 11, middle panels). There are cases when PC1 contributes to predictability but this happens early in the integrations and so is not a result of propagation.

Finally, to examine predictability from a still richer distribution of initial states, we make LIM predictions using each annual mean in the control run as an initial state.

\footnotetext{
${ }^{1}$ Note that in contrast to results for finite ensembles, here relative entropy does converge to zero.
} 
Fig. 10 Same as Fig. 8 but with the LIM simulation results added. We apply the LIM to three different initial conditions taken from the three ensemble experiments. There is only one curve representing the LIM results in $\mathbf{c}$ and $\mathbf{d}$ because the spread of PCs from LIM simulations is independent of the initial state
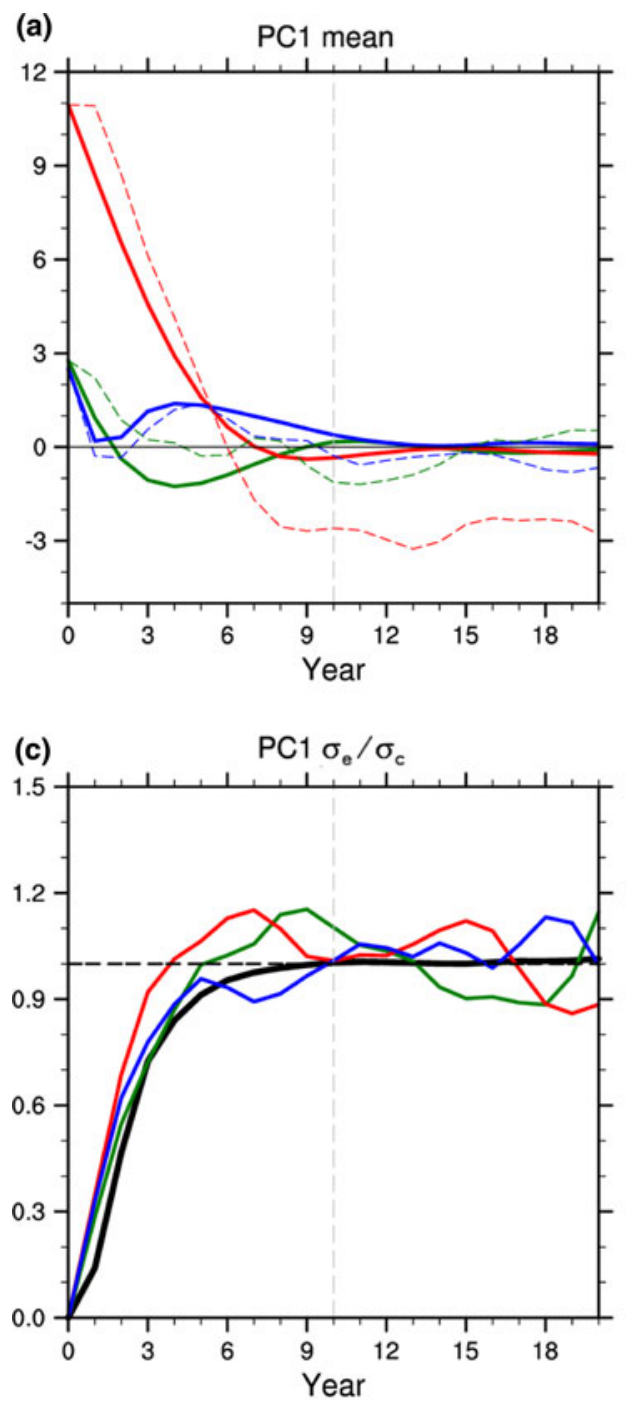
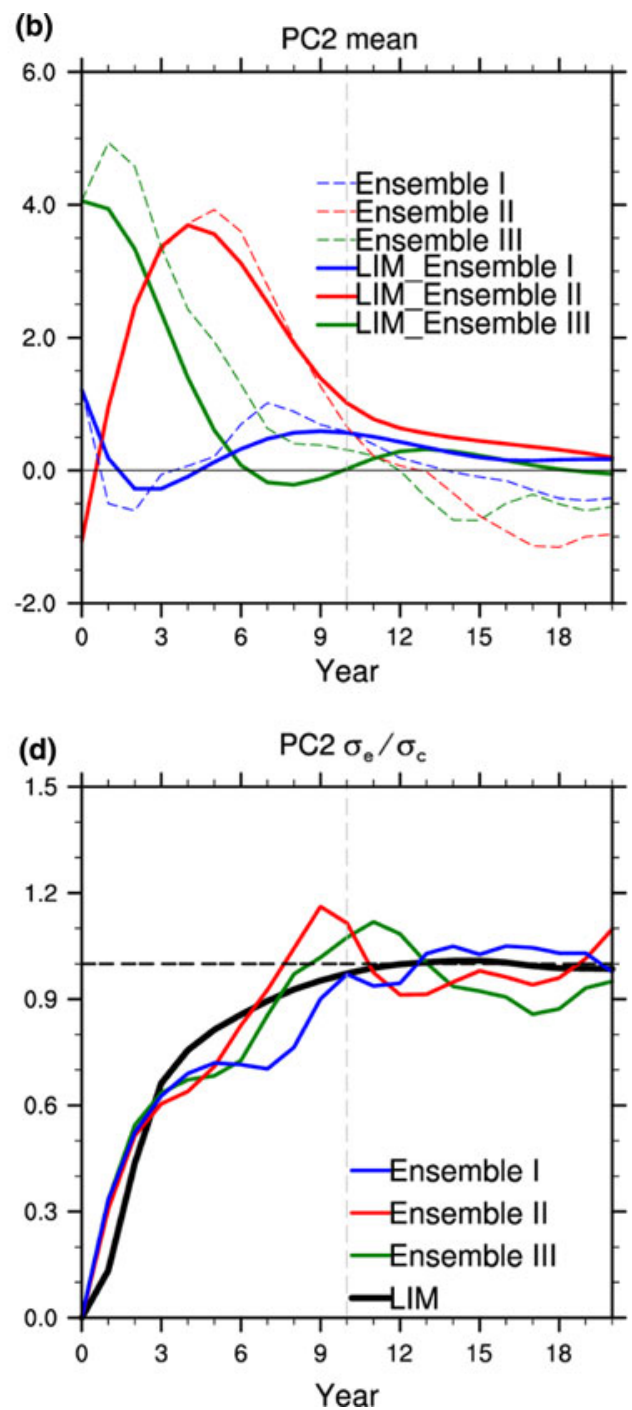

Averaged relative entropy and its components derived from predictions from the 700 initial states is shown in the bottom panel of Fig. 11. It suggests that at the start of the prediction, mean and spread contribute about equally to predictability. But after 3 years for $\mathrm{PC} 1$, and 6 years for PC2, predictability mainly comes from signals. Interestingly, the envelopes of relative entropy in these diagrams indicate that predictability of both PC1 and PC2 may be enhanced by the presence of initial anomalies in subsurface structures other than EOF1 and EOF2. This can be seen, for example at year 6 of PC1, where the most predictable cases out of the 700 tested have relative entropy that is $28 \%$ larger than any of the cases with strong PC1 or PC2.

\section{Comparison to other EOF modes}

After having quantified the predictability limits of the most prominent patterns, now we investigate whether and how much the presence of the two leading EOFs enhances predictability of the North Pacific subsurface temperature. This involves comparing their predictability attributes to those of other major patterns of variability. Again we consider that part of subsurface temperature that is represented by its first 15 EOFs, and we examine each of the CCSM3 ensembles as well as the 700 LIM solutions used in Sect. 5.

We first consider predictability due to spread by using the dispersion component of relative entropy. We find that among the leading 15 EOFs, EOF1 has the fastest growth of spread, while EOF2 and EOF5 have the slowest (Fig. 12; for the sake of clarity, here we only display results for the first five EOFs). This is generally true for all three CCSM3 ensembles as well as for the LIM solutions (all of which have the same spread). This similarity is a further indication that dispersion is not very sensitive to initial conditions. Thus, from the standpoint of spread EOF1 is the least predictable pattern, and it cannot enhance 

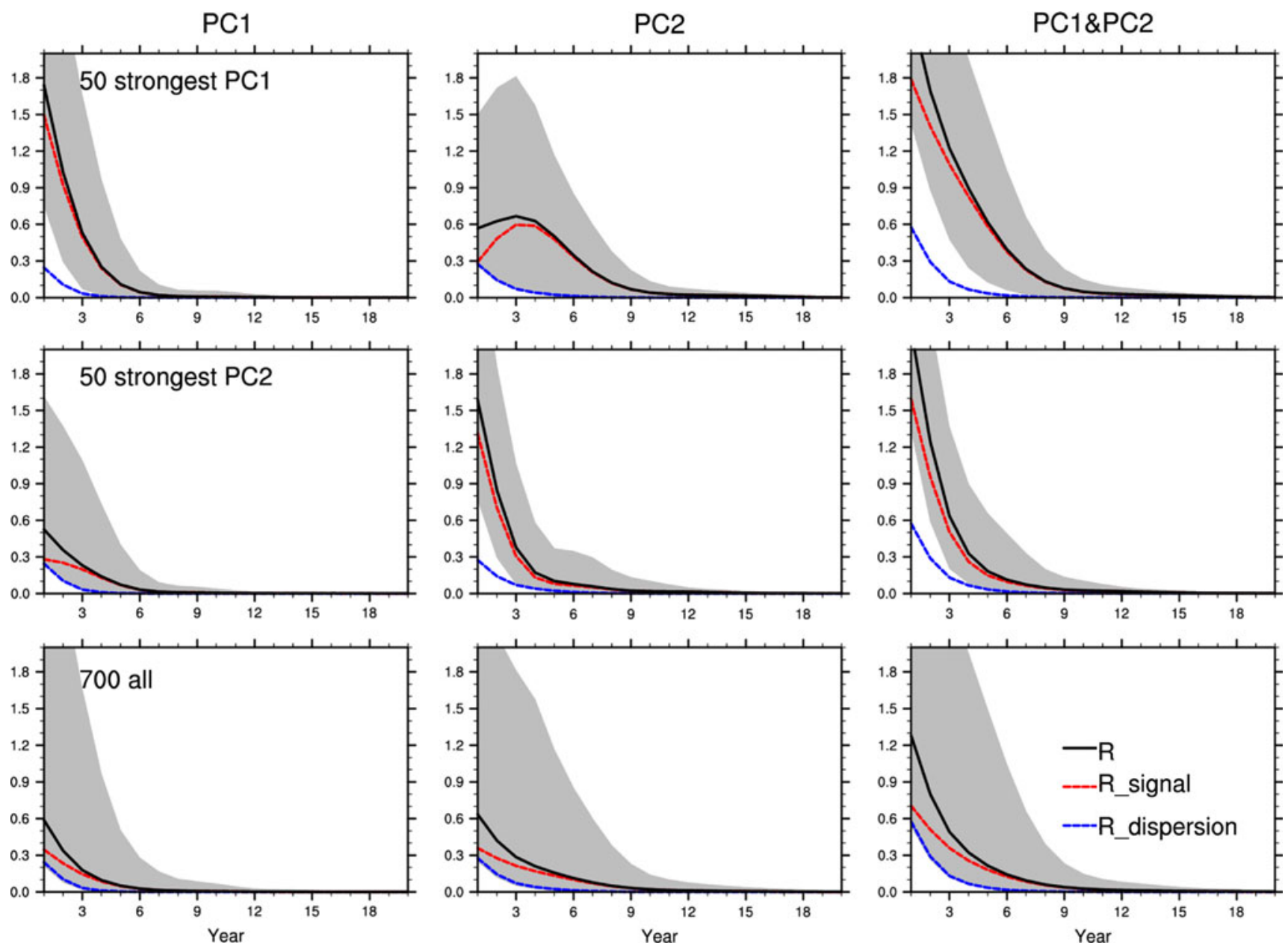

Fig. 11 Univariate relative entropy for PC1 (left) and PC2 (middle) and bivariate relative entropy for PC1 and PC2 combined (right) from three groups of LIM simulations. The first group (top) uses initial conditions associated with the 50 states in the CCSM3 control with the strongest PC1 s. The black, red, and blue lines indicate 50-case averaged total relative entropy, and its signal and dispersion components, respectively, and the grey shading represents the envelope of the 50 cases' total relative entropy. The second group (middle) uses initial conditions associated with the 50 CCSM3 states with the strongest PC2, and the third group (bottom) uses all 700 control states as initial conditions

entropy from the leading two EOFs with that from EOFs 3-15. The sum of the two curves equals the signal component of relative entropy from EOFs 1-15. In contrast to the dispersion component's insensitivity to the initial conditions (Fig. 12), there are large variations among the three ensembles in the signal component. In Ensemble I, the contribution from the first two EOFs is almost zero, and the signal mainly comes from other EOFs. In contrast, EOFs 1-2 explain about $20-30 \%$ of the signal from the leading 15 EOFs during the first 6 years in Ensemble II (Fig. 13b), and during the first 4 years in Ensemble III (Fig. 13c), well above the average contribution from any other two EOFs. So, since the signal component is often such a large part of the predictable information, the degree to which the leading two EOFs contribute to predictability depends strongly on the initial state. But based on the 700 LIM solutions (Fig. 13d), on average these two EOFs make 
Fig. 12 Dispersion component of univariate relative entropy of the leading five EOFs in Ensemble I (a), Ensemble II (b), Ensemble III (c), and from LIM (d)
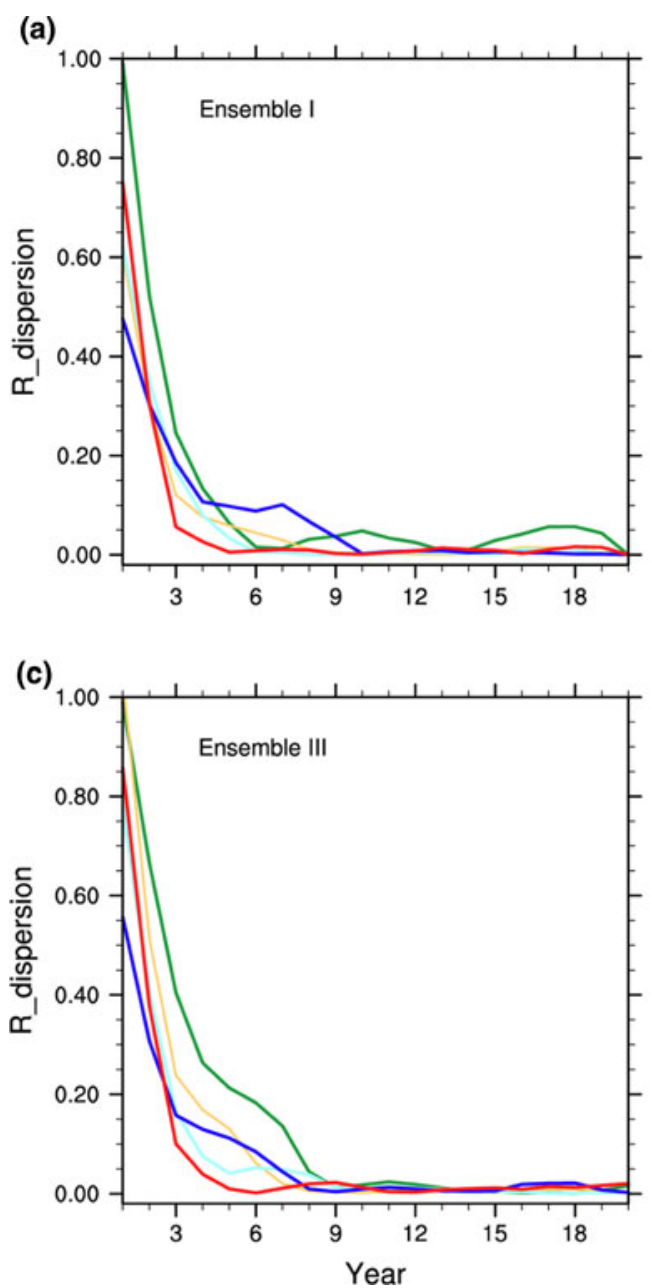

(b)

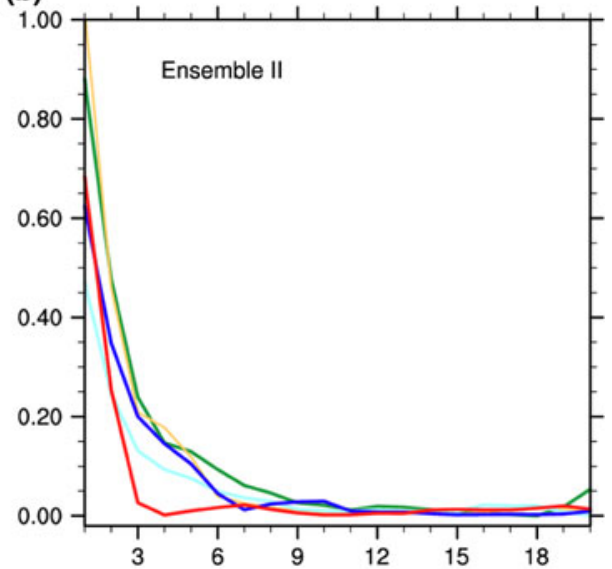

(d)

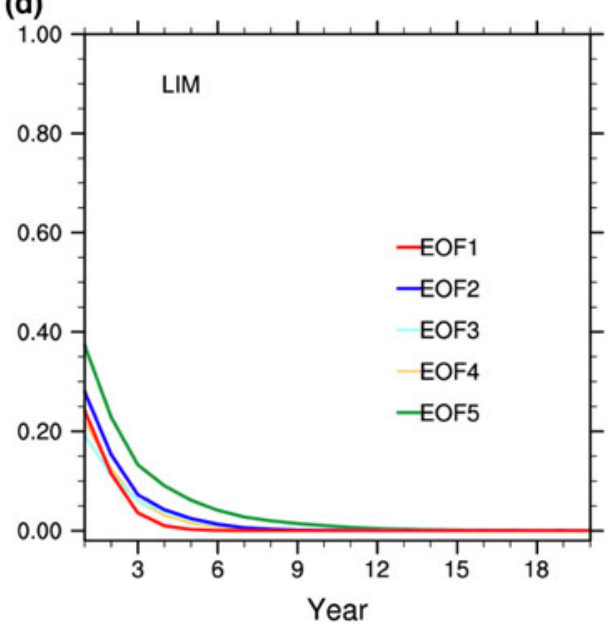

up just $10-20 \%$ of the predictable information during the first five forecast years.

One needs to be cautious of a property of relative entropy when interpreting the top row of Fig. 13. Although relative entropy can measure predictability of a system consisting of several variables, contributions from these variables are given equal weight, as indicated by the normalization of the ensemble means in Eq. 2. And the more variables one considers, the larger the relative entropy will be, so the percentage contributions presented above would be different if we had used a different number of EOFs to describe the temperature field. A modification that is sometimes used to the definition of entropy can avoid this situation. This modification (Karmeshu and Pal 2003) corresponds to including a weighting function in the integrand of Eq. 1 that is a function of $\boldsymbol{x}$. Following this idea, we have recalculated the top row of Fig. 13 with the contribution to the signal term from each PC weighted by the variance of that $\mathrm{PC}$ in the control. This has the effect of removing the division by $\sigma_{c}^{2}$ in Eq. 2. In doing this, we are reasoning that information associated with a variable that tends to have high amplitude is more useful than information associated with low amplitude variables. The results are shown in the bottom row of that figure. As one would expect, with this weighting the contribution to the signal from PC1 and PC2 becomes much more important. Especially in Ensemble II and III, but even for the average LIM case, for at least 6 years the contribution from these two components is comparable to that from the other 13 combined. A second finding of interest that comes from using relative entropy weighted in this way is contained in the grey curve in the bottom panels of Fig. 13. It shows the signal component of weighted relative entropy if one uses 20 EOFs to represent the subsurface temperature state. In all four panels the signal is almost the same as that for 15 EOFs suggesting that when employing 15 EOFs we have included essentially all of the important contributions to information content.

A good example of the motivation behind weighted relative entropy, namely that high predictability in conjunction with large amplitude can lead to a useful forecast, is provided by the propagating mode in Ensemble II. The ensemble averaged subsurface temperature and SST anomalies in year 2, 6, and 8 for this experiment are 
(a)

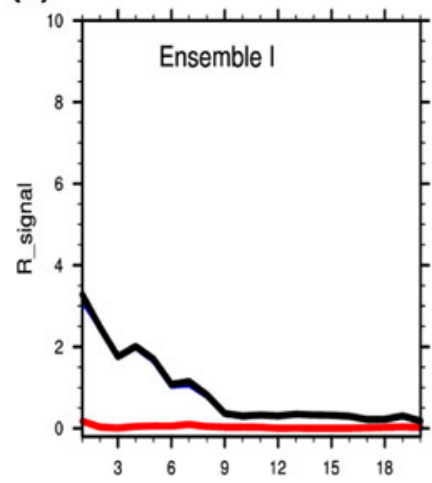

(e)

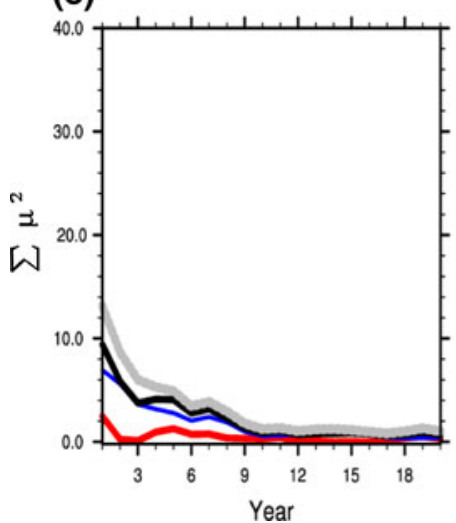

(b)

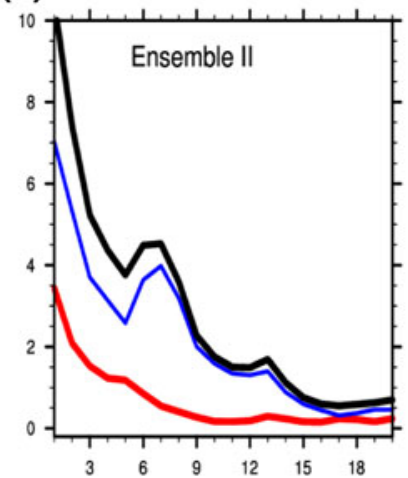

(f)

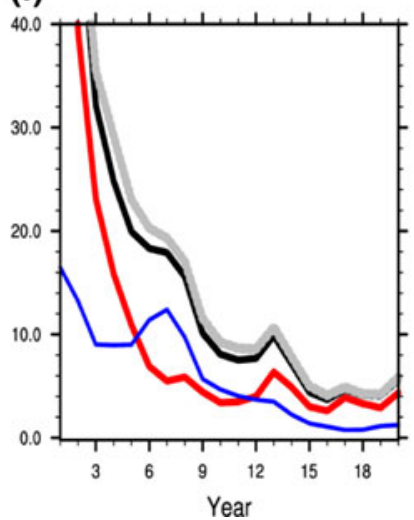

(c)

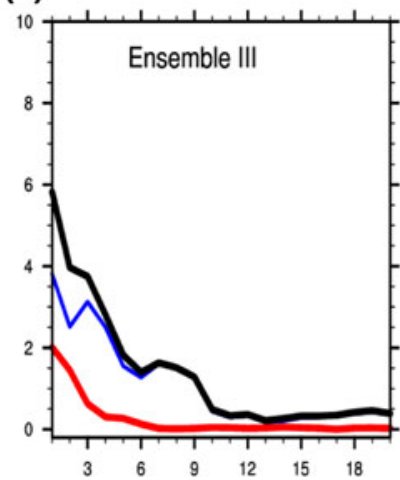

(g)

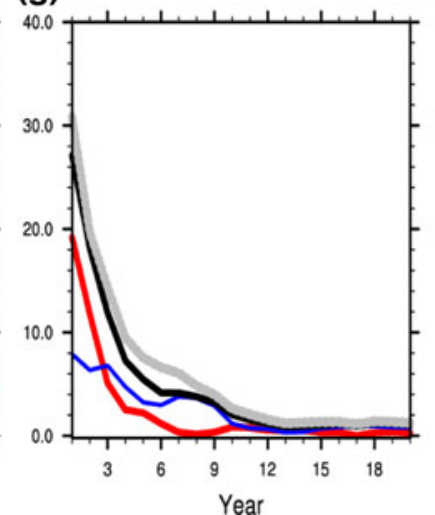

(d)

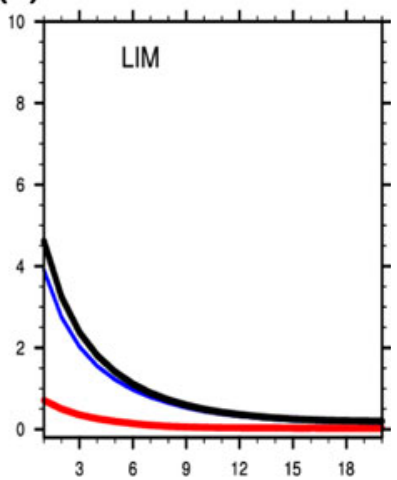

(h)

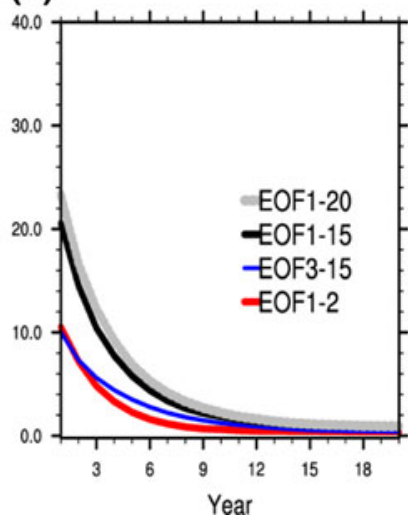

Fig. 13 The top panels are the signal component of relative entropy of the leading 15 EOFs (black), EOF3-15 (blue), and EOF1-2 (red) in Ensemble I (a), II (b), III (c), and for the average of 700 LIM simulations (d). The bottom panels are the same as the top panels but

presented in Fig. 14. These 3 years correspond to phase $32^{\circ}, 95^{\circ}$ and $136^{\circ}$, respectively, of the subsurface propagating mode defined by EOF1 and EOF2. No temporal filtering has been applied to these two fields yet the evolution of both the subsurface temperature and SST resembles the control run composites of filtered fields in Fig. 6 . This indicates that SST anomalies in Ensemble II may be linked to the subsurface mode that has decadal predictability. We also calculate the ensemble average of 10 30 year filtered SLP anomalies for this experiment, and they are shown in the right column of Fig. 14. Similar to the control composite of Fig. 6, during the transitional phase from EOF1 to EOF2 (year 2), North Pacific SLP is governed by positive anomalies north of $50^{\circ} \mathrm{N}$. SLP changes to negative anomalies at year 6 , again in a fashion similar to the corresponding control composite at about phase $90^{\circ}$. Further analysis would be required to establish whether these atmospheric circulation anomalies are caused by the underlying ocean. But the fact that they match relationships seen in the control and the fact that they evolve in a coherent fashion on such long time scales even though intrinsic atmospheric variability on these time scales is typically weak suggests that they may be linked to the signals have not been standardized. Therefore, the signal relative entropy contribution from each EOF is weighted by its explained variance. A grey line for EOF1-20 is also included in (h)

the oceanic mode, which in this case is predictable for about a decade.

\section{Concluding remarks}

While numerous studies have hypothesized that the pronounced decadal variability in the North Pacific, and especially that associated with the PDO, may give rise to decadal predictability, few have carried out experiments specifically designed to measure the predictability of this region and its dominant modes. Here we employ three sets of 40-member ensemble simulations and a 700 year control run from CCSM3 to quantify initial-value predictability in the North Pacific. We focus on the leading subsurface temperature modes and our major results are summarized as follows:

- For average amplitude events, EOF1 (the PDO) is predictable for less than 6 years.

- In terms of ensemble dispersion EOF1 is the least predictable of the leading $15 \mathrm{EOFs}$ while EOF2 is one of the two most predictable. 

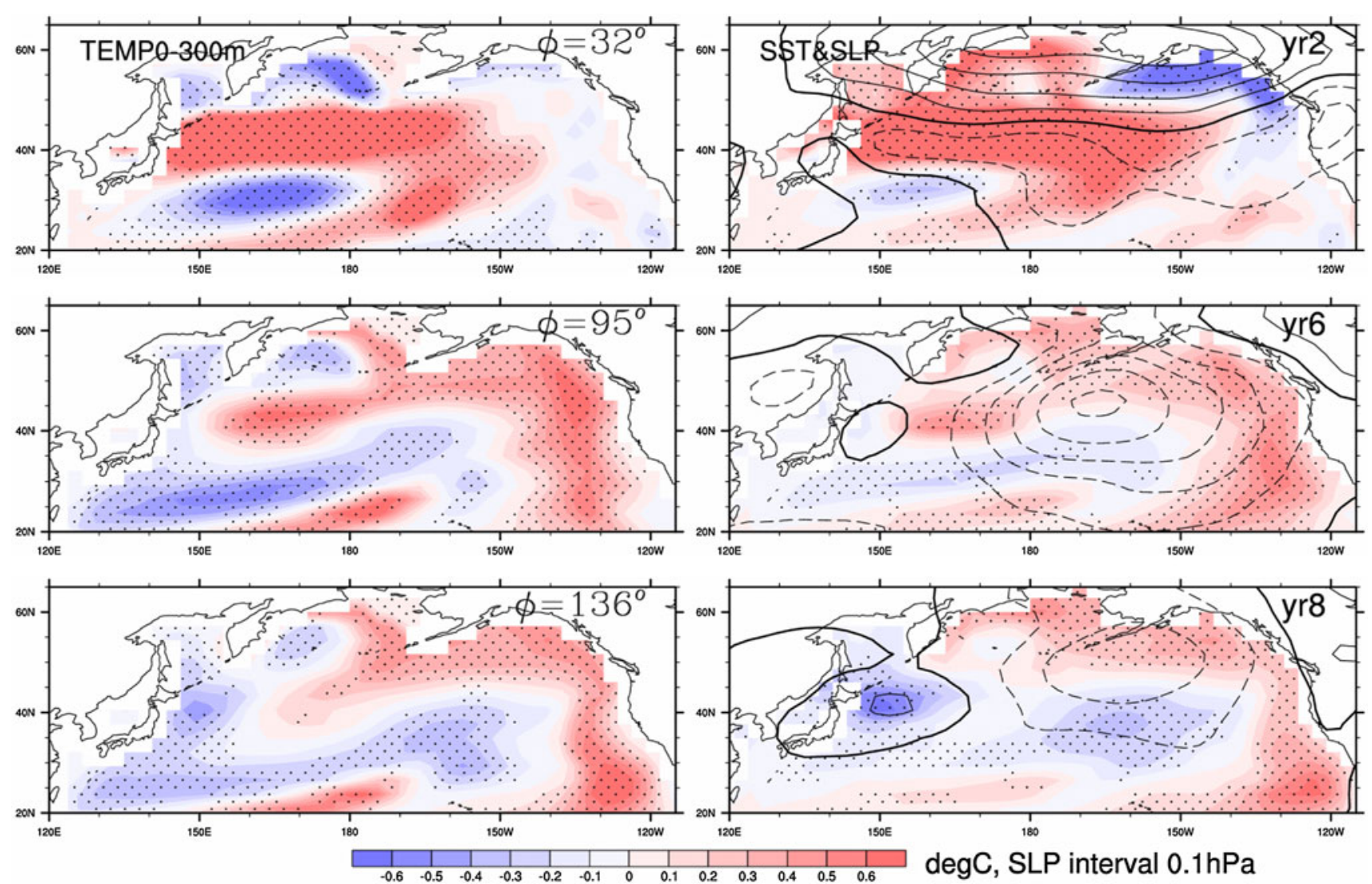

Fig. 14 40-member averaged upper-300 m temperature (left), SST and 10-30 year filtered SLP (right) in year 2 (top), year 6 (middle) and year 8 (bottom) from Ensemble II. Stippling indicates the 95\% significance level from Student's $t$ test

- The leading two EOFs together produce an eastward propagating mode driven by horizontal advection. This mode is not oscillatory; EOF1 tends to evolve to EOF2 but it is much less likely that EOF2 will evolve to (minus) EOF1.

- For initial conditions with average amplitude the propagating mode is predictable for about a decade. Higher predictability results from events beginning with large amplitude PC1 than events beginning with large amplitude PC2.

- For the leading mode information in the ensemble mean tends to be longer lasting than information in the spread about the mean. On average the ensemble spread is indistinguishable from the climatological spread within 5 years while the mean signal lasts nearly a decade. For events with unusually high initial amplitude the mean signal can last even longer.

- The predictability of the leading propagating mode appears to make no larger contribution to basin wide predictability than other patterns of variability from the standpoint of information. But when one considers the high average amplitude of this mode, then its impressive contribution to useful forecasts becomes apparent.
An important lesson from this study is that the intrinsic time scale of a pattern is not necessarily a good indicator of its predictability. We find that PC1 of subsurface temperature has pronounced variance on decadal time scales, but its averaged predictability limit is less than 6 years, perhaps because the atmosphere may be an important contributor to its maintenance and evolution. By contrast, $\mathrm{PC} 2$ has an average predictability limit of about 8 years even though the peaks in its spectrum are at somewhat higher frequencies than those for PC1. This contrast is also seen in the characteristic times of these two PCs: PC1 amplitudes are reduced by a factor of e after 4.5 years while it takes only 3.6 years for such a reduction in the more predictable PC2. The unreliability of time scale as an indicator of predictability is even more dramatic when we consider EOF5. It is the most predictable among the leading 15 EOFs with regard to dispersion rate, yet its e-damping time is 3.5 years. The leading EOF of a geophysical field often possesses the longest intrinsic time scales, and it has been found to have enhanced predictability in studies of weather prediction and intraseasonal variability. Such characteristics have partly motivated us to focus on the leading 
prominent modes as a first step toward quantifying predictability in the North Pacific. But our results here provide a counterexample to a correspondence between time scales of a pattern and its predictability and indicate one must take a prognostic approach in order to get an accurate estimate of predictability limits.

A second clear lesson from our investigation is the need to allow for time evolving modes when examining predictability. EOF1 appears to have low predictability when considered in isolation. But this conclusion changes when one takes into account its tendency to evolve to EOF2, which is one of the most predictable patterns. Interestingly it is also apparent that the initial phase of such time dependent modes must be accounted for when quantifying their predictability.

We are uncertain which physical process determines the 20 year dominant frequency peak of the prominent mode captured by EOF1 and EOF2. Our result that the dominant eastward propagation of this mode is mainly caused by horizontal advection does not eliminate the possibility suggested by some studies (Jin 1997; Qiu 2003) that baroclinic Rossby waves resulting from oceanatmosphere interactions may play a role in timescale selection of prominent North Pacific modes. In results not displayed in this paper, we have found suggestions of westward propagating features in sea surface height to the north of the eastward propagating features we have focused on. In addition, we find hints of anomalies propagating southwestward along the subtropical subduction pathway in composites of subsurface temperature, but they don't propagate all the way to the western boundary. Our main point, from the perspective of predictability, is that in CCSM3 westward propagating features are not prominent enough to sustain full oscillations as exemplified by the fact that in the CCSM3 ensemble experiments and in the LIM, EOF2 does not necessarily lead to growth of EOF1.

A third important lesson from our work is that both the mean and spread of an ensemble should be taken into account when assessing initial-value predictability. A common question for predictability studies is whether there exist some initial ocean states that are more predictable than others. In contrast to common perceptions that ensemble spread is the primary factor that distinguishes the most predictable situations (Palmer 1993), all three of our ensembles show similar rates of spread; the longest predictability is found in the ensemble with an especially strong initial anomaly that leads to a long lasting mean signal. This behavior resembles the predictability characteristics of a stochastically forced damped linear system (Kleeman 2002). The fact that we were able to replicate the statistics of the prominent propagating mode in the three ensembles rather well by a LIM further implies that the
PDO in CCSM3 may to a large extent be approximated as a stochastic, damped mode.

Beyond these results and their implications, our work adds to previous predictability studies in the following ways: First, we focus on subsurface temperature. Our results suggest that the subsurface propagating mode, which has decadal predictability, is associated with signatures in SST and atmospheric surface pressure. This finding opens the possibility that filtering surface conditions to retain anomalies that are associated with predictable subsurface structures might be a way of isolating those components of surface conditions that are most predictable on decadal time scales. Second, our predictability limit is estimated using relative entropy, which can assess the forecast range at which information present in the initial condition is lost based on all aspects of a predicted distribution. An additional benefit of relative entropy is that it can measure information flow in a multivariate distribution making it ideal for considering modes with more than one spatial degree of freedom. Methodologies based only on isolated geographical locations or single patterns, e.g. univariate AR1 modeling, would be misleading under these circumstances.

Though we believe the various implications of our work are worthwhile, it is best to also keep in mind various limitations to the conclusions that should be drawn from our results because of our experimental design and model errors. Perhaps paramount among these is that just because we have measured the predictability of certain modes does not mean that this predictability can be attained; it is only an upper bound on what can be attained. Furthermore the predictability limits we report above are only valid for CCSM3. Other models and nature may well have different limits.

Beyond these unavoidable shortcomings in our investigation, there are others gaps in our study that can be addressed through additional work. Our current study has focused on prominent modes that explain $40 \%$ of the total variance. It would be worthwhile to expand the analysis from individual modes to generic forecast fields. Second, investigations in other ocean basins would be useful. In preliminary work, we have calculated the RMSD of SST and subsurface temperature in several ocean basins and find the saturation time is highly dependent on region. Figure $1 \mathrm{~b}$ gives one example of this, namely the equatorial Pacific, where saturation is reached much earlier than in the North Pacific. Third, though we have shown that the subsurface propagating mode carries a signature in SST and atmospheric surface pressure, it would be useful to quantify the predictability of this component of low-frequency SST and atmospheric variability. Fourth, we have not investigated the structure of those perturbations that lead to the fastest loss of predictability though such information is 
needed for designing the climate observational system. Fifth, we have only considered predictability of the first kind while there is a forecast range at which predictability of the second kind should dominate (Hawkins and Sutton 2009; Meehl et al. 2009). We expect to consider these topics in future investigations.

Acknowledgment The authors acknowledge many colleagues for useful conversations and support from the DOE under Cooperative Agreement No. DE-FC02-97ER62402. NCAR is sponsored by the National Science Foundation.

\section{References}

Alexander M, Yin J, Branstator G, Capotondi A, Cassou C, Cullather R, Kwon YO, Norris J, Scott J, Wainer I (2006) Extratropical atmosphere-ocean variability in CCSM3. J Clim 19:2496-2525

Alexander M, Blade I, Newman M, Lanzante J, Lau NC, Scott JD (2002) The atmospheric bridge: the influence of ENSO teleconnections on air-sea interaction over the global oceans. J Clim 15:2205-2231

Alexander M, Matrosova L, Penland C, Scott JD, Chang P (2008) Forecasting Pacific SSTs: linear inverse model predictions of the PDO. J Clim 21:385-402

Boer GJ (2000) A study of atmosphere-ocean predictability on long time scales. Clim Dyn 16:469-477

Boer GJ (2004) Long time-scale potential predictability in an ensemble of coupled climate models. Clim Dyn 23:29-44

Branstator G, Mai A, Baumhefner D (1993) Identification of highly predictable flow elements for spatially filtering of medium- and extended-range numerical forecasts. Mon Weather Rev 121:1786-1802

Bryan FO, Danbasoglu G, Nakashiki N, Yoshida Y, Kim DH, Tsutsui J, Doney SC (2006) Response of the North Atlantic thermohaline circulation and ventilation to increased carbon dioxide in CCSM3. J Clim 19:2382-2397

Collins M (2002) Climate predictability on interannual to decadal time scales: the initial value problem. Clim Dyn 19:671-692

Collins M, Botzet M, Carril AF, Drange H, Jouzeau A, Latif M, Masina S, Otteraa OH, Pohlmann H, Sorteberg A, Sutton R, Terray L (2006a) Interannual to decadal climate predictability in the North Atlantic: a multimodel-ensemble study. J Clim 19:1195-1203

Collins WD, Bitz CM, Blackmon ML, Bonan GB, Bretherton CS, Carton JA, Chang P, Doney SC, Hack JJ, Henderson TB, Kiehl JT, Large WG, McKenna DS, Santer BD, Smith RD (2006b) The community climate system model version 3 (CCSM3). J Clim 19:2122-2143

Collins M, Sinha B (2003) Predictability of decadal variations in the thermohaline circulation and climate. Geophs Res Lett 30. doi: 10.1029/2002GL016504

Deser C, Alexander M, Timlin MS (2003) Understanding the persistence of sea surface temperature anomalies in midlatitudes. J Clim 16:57-72

Frankignoul C, Hasselmann K (1977) Stochastic climate models. Part II: application to sea-surface temperature anomalies and thermocline variability. Tellus 29:289-305

Frankignoul C, Muller P, Zorita E (1997) A simple model of the decadal response of the ocean to stochastic wind forcing. J Phys Oceanogr 27:1533-1546

Griffies SM, Bryan K (1997a) A predictability study of simulated North Atlantic multidecadal variability. Clim Dyn 13:459-488
Griffies SM, Bryan K (1997b) Predictability of North Atlantic multidecadal climate variability. Science 275:181-184

Hawkins E, Sutton R (2009) The potential to narrow uncertainty in regional climate predictions. Bull Am Meteorol Soc (in press)

Hurrell JW et al (2009) Decadal climate prediction: opportunities and challenges. Community White Paper, OceanObs 2009

Jin F-F (1997) A theory of interdecadal climate variability of the North Pacific ocean-atmosphere system. J Clim 10:1821-1835

Karmeshu A, Pal NR (2003) Uncertainty, entropy and maximum entropy principle: an overview. In: Karmeshu (ed) Entropy measures, maximum entropy principle and emerging applications. Springer, Berlin, pp 1-78

Keenlyside N, Latif M, Junclaus J, Kornblueh L, Roeckner E (2008) Advancing decadal climate scale prediction in the North Atlantic. Nature 453:84-88

Kleeman R (2002) Measuring dynamical prediction utility using relative entropy. J Atmos Sci 59:2057-2072

Kwon YO, Deser C (2007) North Pacific decadal variability in the community climate system model version 2. J Clim 20:24162433

Latif M, Barnett TP (1994) Causes of decadal climate variability over the North Pacific and North America. Science 266:634-637

Latif M, Barnett TP (1996) Decadal climate variability over the North Pacific and North America: dynamics and predictability. J Clim 9:2407-2423

Lorenz EN (1963) Deterministic non-periodic flow. J Atmos Sci 20:130-141

Mantua NJ, Hare SR, Zhang Y, Wallace JM, Francis RC (1997) Pacific interdecadal climate oscillation with impacts on salmon production. Bull Am Meteorol Soc 78:1069-1079

Meehl GA, Washington WM, Santer BD, Collins WD, Arblaster JM, $\mathrm{Hu}$ A, Lawrence DM, Teng H, Buja L, Strand WG (2006) Climate change projections for the twenty-century and climate change commitment in the CCSM3. J Clim 19:2597-2626

Meehl GA, Goddard L, Murphy J, Stouffer RJ, Boer G, Danabasoglu G, Dixon K, Giorgetta MA, Greene AM, Hawkins E, Hegerl G, Karoly D, Keenlyside N, Kimoto M, Kirtman B, Navarra A, Pulwarty R, Smith D, Stammer D, Stockdale T (2009) Decadal prediction: can it be skillful? Bull Am Meterol Soc 90:14671485

Miller AJ, Schneider N (2000) Interdecadal climate regime dynamics in the North Pacific ocean: theories, observations and ecosystem impacts. Prog Oceanogr 47 Pergamon, 355-379

Newman M (2007) Interannual to decadal predictability of tropical and North Pacific sea surface temperatures. J Clim 20:2333-2356

Newman M, Sardeshmukh PD, Winkler CR, Whitaker JS (2003a) A study of subseasonal predictability. Mon Weather Rev 131:1715-1732

Newman M, Compo GP, Alexander MA (2003b) ENSO forced variability of the Pacific decadal oscillation. J Clim 16:38533857

North GR, Bell TL, Cahalan RF, Moeng FJ (1982) Sampling errors in the estimation of empirical orthogonal functions. Mon Weather Rev 110:699-706

Palmer TN (1993) Extended-range atmospheric prediction and Lorenz model. Bull Am Meteorol Soc 74:49-65

Penland C (1989) Random forcing and forecasting using principal oscillation pattern analysis. Mon Weather Rev 117:2165-2185

Penland C, Sardeshmukh PD (1995) The optimal growth of tropical sea surface temperature anomalies. J Clim 8:1999-2024

Pohlmann H, Botzet M, Latif M, Roesch A, Wild M, Tschuck P (2004) Estimating the decadal predictability of a coupled AOGCM. J Clim 17:4463-4472

Qiu B (2003) Kuroshio extension variability and forcing of the Pacific decadal oscillation: responses and potential feedback. J Phys Oceanogr 33:2465-2482 
Saravanan R, McWilliams JC (1998) Advective ocean-atmosphere interaction: an analytical stochastic model with implications for decadal variability. J Clim 11:165-188

Seager R, Kushnir Y, Naik N, Cane MA, Miller JA (2001) Winddriven shifts in the latitudeof the Kuroshio-Oyashio extension and generation of SST anomalies on decadal timescales. J Clim 14:4249-4265

Smith D, Cusack S, Colman AW, Folland CK, Harris GR, Murphy JM (2007) Improved surface temperature prediction for the coming decade from a global climate model. Science 317:796-799
Tribbia J (1988) The predictability of monthly mean teleconnection patterns. Study week on: persistent meteo-oceanographic anomalies and teleconnections. Vatican City, Pontificia Academia Scientiarum, 567-592

von Storch H, Zwiers FW (1999) Statistical analysis in climate research. Cambridge University Press, Cambridge

Zhong Y, Liu Z, Jacob R (2008) Origin of Pacific multidecadal variability in Community Climate System Model, version 3 (CCSM3): a combined statistical and dynamical assessment. J Clim 21:114-133 